\title{
Airborne quantum key distribution: a review [Invited]
}

\author{
Yang Xue (薛 阳) $)^{1,2, \dagger}$ ，Wei Chen (陈 巍 $)^{1^{1,}, \dagger}$, Shuang Wang (王 双) ${ }^{1}$, Zhenqiang Yin (银振强) $)^{1}$, Lei Shi (石 否 $)^{2}$, and \\ Zhengfu Han (韩正甫) $)^{\text {t** }}$ \\ ${ }^{1}$ CAS Key Laboratory of Quantum Information, University of Science and Technology of China, Hefei 230026, China \\ ${ }^{2}$ Information and Navigation College, Air Force Engineering University, Xi'an 710077, China
}

*Corresponding author: weich@ustc.edu.cn

${ }^{\star *}$ Corresponding author: zfhan@ustc.edu.cn

Received July 2, 2021 | Accepted September 29, 2021 | Posted Online November 3, 2021

\begin{abstract}
Remarkable achievements have been witnessed in free-space quantum key distribution (QKD), which acts as an available approach to extend the transmission range of quantum communications. The feasibility of transmitting qubits through the free-space channel with the aid of moving platforms like satellites, aircraft, unmanned aerial vehicles (UAVs) has been verified. In view of the limited working time and resource consumption of the satellite-based QKD and the last-mile challenges of connecting satellite nodes with terrestrial networks, the airborne QKD is expected to provide flexible and relay links for the large-scale integrated network. This paper reviews the recent significant progress of QKD based on aircraft or UAVs, highlights their critical techniques, and prospects the future of airborne quantum communications.
\end{abstract}

Keywords: quantum communication; airborne quantum key distribution.

DOI: 10.3788/COL202119.122702

\section{Introduction}

The unique properties of qubits governed by quantum mechanics laws provide us with new methods for secure communication with unparalleled information processing capability ${ }^{[1,2]}$. As one of the most mature branches in quantum information technology, quantum key distribution $(\mathrm{QKD})^{[3-6]}$ ensures the secure generation of symmetric keys between distant parties relying on physical principles rather than computational assumptions. The users can combine the key bits with the encryption method like one-time-pad (OTP) ${ }^{[7]}$ or advanced encryption standard $(\mathrm{AES})^{[8]}$ to guarantee information security. QKD has shown its great potential in coping with the unanticipated threat posed by the improvement of hardware and more efficient algorithms, including quantum computing ${ }^{[9-11]}$.

The ultimate goal of quantum communication is to establish a global quantum Internet ${ }^{[12,13]}$. As the roadmap has pointed out ${ }^{[13]}$, establishing global QKD networks are the primary stage. More than three decades of theoretical and experimental research efforts have been made since the heuristic 1984 Bennett-Brassard (BB84) protocol was proposed ${ }^{[3]}$, making $\mathrm{QKD}$ leap out of the laboratory and march towards diverse real-life applications ${ }^{[14]}$. For instance, it has been employed to encrypt the ballot data in Switzerland's national election ${ }^{[15]}$, to provide high-level information security in local governmental networks ${ }^{[16-19]}$, and even achieve the private video call between Beijing and Vienna ${ }^{[20]}$.

Although QKD research has made remarkable progress ${ }^{[21]}$, there are still two major challenges to achieve realistic secure networks. One is the significant gap between theoretic models and realistic devices, compromising the practical systems' performances. The other lies in the difficulty of building high-rate and stable links for qubit transmission over long distances and broad areas.

Photons are the natural physical carriers of quantum information since they are convenient to be prepared, manipulated, and transferred ${ }^{[22]}$. Therefore, the fiber and the free-space channel are the optimal quantum channels up to date. The final key rate of QKD decays rapidly in fiber when extending the transmission distance due to the channel attenuation and detector noise. Various QKD protocols have been proposed to beat the fundamental ratedistance bound ${ }^{[23-30]}$, and the attainable range of QKD has been over $500 \mathrm{~km}$ using the ultra-low-loss fiber ${ }^{[31,32]}$.

There is a consensus that the future global quantum network cannot be realized depending on a single scheme ${ }^{[33,34]}$. Free-space QKD plays a vital role in building a secure global quantum communication network. Bennett et al. demonstrated the first proof-of-principle QKD experiment in a $32 \mathrm{~cm}$ freespace channel ${ }^{[35]}$. Hughes et al. completed the free-space QKD experiments over $1 \mathrm{~km}$ and $10 \mathrm{~km}$ free-space channels in 1998 and 2002, respectively ${ }^{[36,37]}$. Soon after, Kurtsiefer et al. accomplished a field experiment of BB84 QKD in a $23.4 \mathrm{~km}$ freespace link between two mountains ${ }^{[38]}$. In 2007, SchmittManderbach et al. extended the free-space QKD link to $144 \mathrm{~km}$ between two islands adopting the decoy-state method ${ }^{[39]}$. Recently, the Chinese quantum satellite Micius has fulfilled 
a series of demonstrations of fundamental quantum physics and QKD protocols at space-bound large-scale separations, which marked a milestone in free-space quantum communication researches ${ }^{[40-42]}$.

At present, most of the terrestrial free-space QKD experiments are performed in stationary line-of-sight links. Although satellites are good candidates for providing QKD service over $1000 \mathrm{~km}$ or even at the intercontinental scale, daunting costs and limited operation time windows of satellites hinder their massive deployment.

Due to the line-of-sight propagation feature of photonic signals, the free-space links cannot bypass physical obstructions such as mountains, buildings, and trees. Besides, these links may be blocked by interference like fog and clouds. Therefore, establishing the mobile nodes and links as demanded is essential to satisfy the requirement of practical QKD applications.

Airborne platforms or high-altitude platforms (HAPs) are ideal mobile nodes that can synergize with terrestrial links and quantum satellites to build a global quantum network. This review discusses QKD and entanglement distribution systems based on the aircraft in free-space channels. First, we introduce the basic concept and progress of airborne QKD experiments. Second, the salient advantages and critical techniques of airborne quantum communications are summarized. The third part highlights the future challenges and perspectives, and we conclude in the final part.

\section{Concept and Progress}

In this section, we focus on the basic concepts and recent progress of airborne QKD. We also discuss the advantages of adopting the airborne platform for quantum communications.

\subsection{Basic concept}

Airborne QKD refers to specific schematics to achieve quantum communication in free-space targeting short-range, local-area networks, and mobile scenarios. Compared with terrestrial free-space experiments, airborne QKD features dynamic optical links due to drastic relative motions between the transmitter and receiver. Figure 1 shows the blueprint of a hierarchical quantum network operating in different atmospheric layers. The satellite QKD aims at establishing quantum channels at the scale of thousands of kilometers. Generally, a lower altitude satellite benefits from lower diffraction-induced losses and launching cost. However, the line-of-sight timing window will be shorter, and the acquisition, tracking, and pointing (ATP) system should be more accurate and faster. Hence, the communication efficiency, duration, complexity, and cost should be balanced with synthetic considerations, and all available technologies should be optimally integrated to build the global quantum network.

Similar to the satellite QKD, diverse flying vehicles have different characteristics and their optimum application scenarios. The physical height limit of a drone with spinning propellers is around $10 \mathrm{~km}$. However, the off-the-shelf drones commonly fly

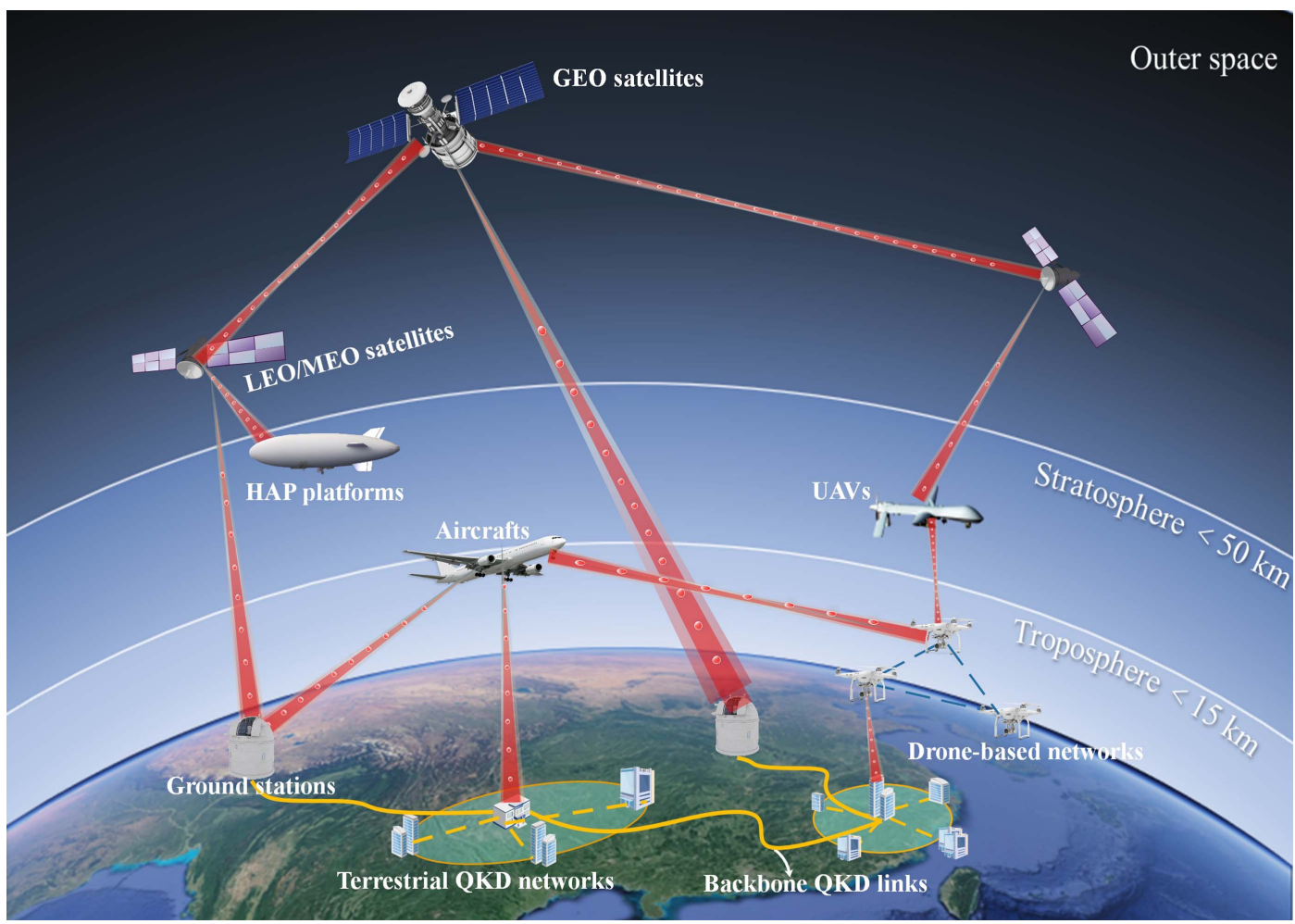

Fig. 1. Hierarchical quantum network operating in different atmospheric layers. LEO, low Earth orbit; MEO, medium Earth orbit; GEO, geostationary Earth orbit; HAP platform, high-altitude platform; UAV, unmanned aerial vehicle. 
below $500 \mathrm{~m}$ under normal conditions. The moving platforms like the manned/unmanned aircraft and helicopters fly in the region of 5-15 km, while the floating vehicles such as hot-air balloons and HAPs work above $15 \mathrm{~km}^{[43]}$.

Airborne quantum nodes like drones or unmanned aerial vehicles (UAVs) could serve as temporary relays to solve the last-mile quantum key exchange for an inner-city or a field network benefiting from their rapid deployment capabilities. For higher altitude, the low-velocity aircraft is advantageous to provide longer link duration and broader transmission coverage. Quantum communication based on this platform can serve as a tactical approach to secure confidential data at a larger scale. Beyond the height of the troposphere, airborne platforms can assist in relaying the quantum signals from satellites to the ground or vice versa.

In general, airborne systems present a flexible approach for expanding the scope of quantum communications in time and space. Within the context of intercontinental satellite links, which have been verified ${ }^{[20]}$, airborne quantum communication can be a beneficial supplement to the strategy of global secure information transfer networks with a relatively low cost and high flexibility.

\subsection{Recent progress on airborne quantum communications}

Several groups have reported their research results in airborne quantum communications in recent years, including the airto-ground or backlink QKD between a rapidly moving aircraft and a ground station ${ }^{[44,45]}$, as well as the direct and full-scale quantum experiments using the hot-air balloon ${ }^{[46]}$. Some projects even envision using airships and fixed-wing HAPs as relay stations for key distribution due to their flexibility, endurance, payload capacity, and easy maintenance ${ }^{[47]}$. Notably, a dronebased quantum entanglement distribution has been demonstrated in multi-weather conditions ${ }^{[48]}$ and optical-relayed entanglement distribution ${ }^{[49]}$, which laid solid foundations in establishing reconfigurable mobile quantum networks. Figure 2 shows the research timeline of airborne quantum communications and the different mobile platforms they utilized in the recent decade.

In the view of recent experiments, airborne platforms are primarily used to simulate the altitude variations and relative motions of a satellite to evaluate the feasibility of spaceborne QKD sessions. However, with the rapid growth of the UAV industry and the development of automatic control technologies, UAVs or drones have become popular in the military, rescue, metrology, and even logistics and delivery ${ }^{[55]}$. They have also found a wide range of applications in wireless communication and free-space optics (FSO) communication due to their outstanding performance. For example, integrating the UAVbased nodes into a macro cell network has been considered a critical supplementary solution for terrestrial cellular networks supporting secure $5 \mathrm{G}$ communication ${ }^{[56]}$. At the same time, the idea of building a reconfigurable mobile QKD network has been proposed and analyzed in theory ${ }^{[57]}$. In principle, the drone can be a prospective selection to fulfill this task. It shows a flexible
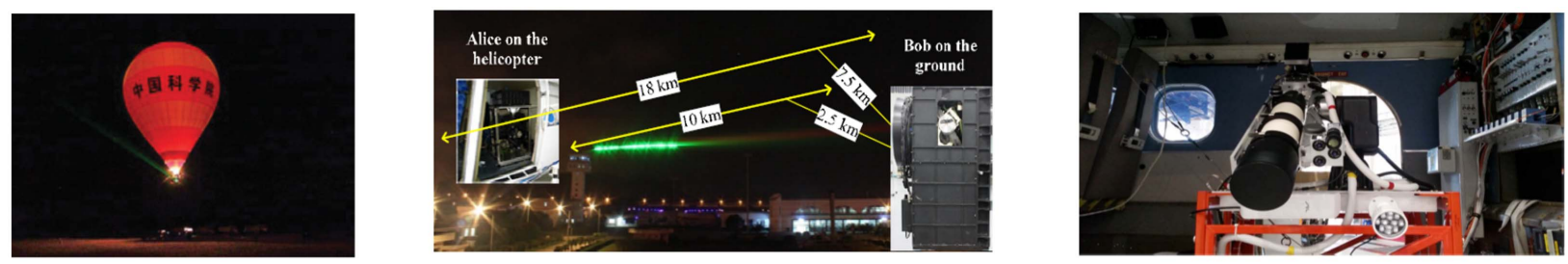

- USTC

- Hot-air balloon
- CAS - Z-9 helicopter
- University of Waterloo

- Twin Otter
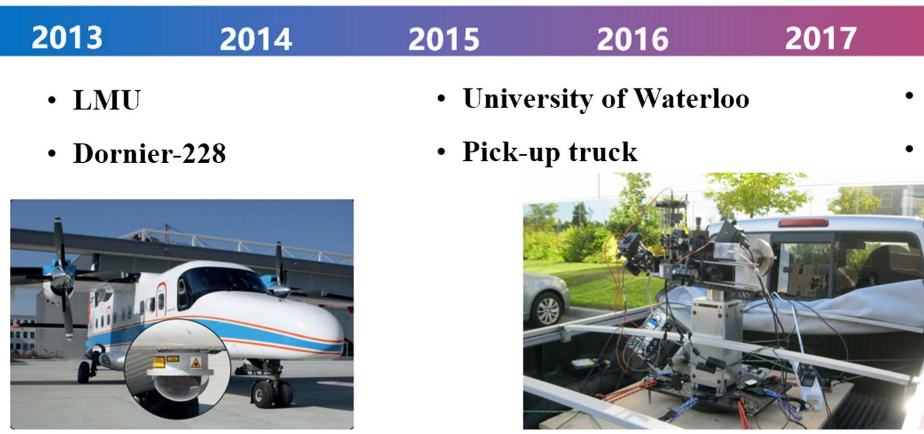

$2018 \quad 2019$

2020

- Nanjing University

- DJI S1000 + Octocopter
- DJI Phantom 4

Fig. 2. Recent progress in airborne quantum communications. In clockwise order, the first downlink QKD demonstration in 2013 using the hot-air balloon by Wang et al. ${ }^{[46]}$, the basis detection and compensation experiment in 2014 using the Z-9 helicopter by Zhang et al. from the Chinese Academy of Sciences ${ }^{[50]}$, the first uplink QKD demonstration in 2017 using the Twin Otter research aircraft by Pugh et al. from the University of Waterloo ${ }^{[45]}$, the first drone-based entanglement distribution in 2020 using UAV by Liu et al. from the Nanjing University ${ }^{[48,49]}$, the drone-based QKD test in 2017 using DJI S1000+ octocopter by Hill et al. from the University of Illinois ${ }^{[51-53]}$, the free-space QKD in 2015 based on a moving pick-up truck by Bourgoin et al. from the University of Waterlo[ ${ }^{[54]}$, and the first air-toground QKD demonstration in 2013 using the Dornier-228 aircraft by Nauerth et al. from the Ludwig-Maximilians University ${ }^{[44]}$. 
and cost-effective way to extend the range of secure communication and provides real-time coverage on demand. These features enable quantum cryptography to adapt to diverse application scenarios, such as the temporarily deployed secure networks in seaborne, urban, or battlefield situations.

What is more, Vasylyev et al. investigated the statistical features of quantum light to account for the influence of atmospheric turbulence and random scattering caused by raindrops ${ }^{[58]}$. Their theory will be beneficial to the system design and realization of airborne QKD in severe weather. Besides, the flexible establishing link of airborne QKD can make an optimal performance by adjusting its flying parameters according to the environments, while taking advantage of the free-space QKD.

Table 1 shows a summary of the recent progress of airborne QKD and related airborne projects. The following section will discuss the critical technologies implementing airborne QKD and discuss the issues that may need to be improved in the next step.

\subsection{Advantages of applications}

Airborne platforms can provide a unique environment to explore the boundaries of quantum theory in aeronautical environments with microscopic particles or atmospheric factors, which are inaccessible in the laboratory ${ }^{[60-63]}$. Furthermore, from the perspective of real-life applications, airborne QKD can enhance the security of wireless communication as an auxiliary approach of the existing aeronautical communications like radio frequency $(\mathrm{RF})$ and FSO. Moreover, it is an effective way to establish a mobile secure communication network in a temporary, emergency, or fiber-less condition. Lastly, it can play the role of trusted relays to enhance links between the satellite and ground-based users in the global-scale quantum network infrastructure.

The most important advantage of the airborne QKD lies in its high deployment flexibility and maneuverability. Aircrafts require no existing fiber channel and are not restricted by predictable trajectories and complicated launching activities compared with satellites. Consequently, the reusability and long endurance of aircrafts allow the QKD links to be deployed in remote areas on demand and have promise in extending the available working duration in cooperation with the satellitebased QKD.

The next advantage of airborne QKD is robustness against diverse weather conditions. In order to realize a wide-area allweather quantum communication network, airborne platforms should avoid being blocked by clouds, haze, or terrible weather by traveling to the sites that are not affected by the weather. The mobility of the airborne platform can assist the system in searching a link with lower background noises. To this end, Liu et al. tested the S-parameter of the Clauser-Horne-Shimony-Holt (CHSH-S) inequality in their drone-based entanglement distribution experiment during clear daytime, clear night, and rainy night ${ }^{[48]}$. The current link distance is about $200 \mathrm{~m}$, and extending the distance to tens of kilometers in poor weather conditions is still a formidable technical challenge up to date. However, these experimental results show the possibilities of achieving full-weather quantum communications.

In general, there are two ways to help airborne QKD extend its communication distance and cope with more complex channel conditions. The first one is to utilize the relatively stable flying platforms, which have better loading capacities, and then more complex optical devices with larger apertures and adaptive optics can be adopted. Platforms like hot-air balloons or airplanes have shown the potential to carry out real-life QKD over the channel distance of about $20 \mathrm{~km}$, as shown in Table 1 . Secondly, it is possible to cover a wider area or tolerate more complex weather conditions by integrating numbers of airborne nodes into a network as relays or terminals. This scheme has been demonstrated by Liu et al. with the experiment of entanglement distribution ${ }^{[49]}$. The former approach may offer more stable and durable links. However, the system may be more complex, and the relatively fixed links have no detour for a versatile channel. The latter is more robust and flexible in finding circuitous routes to avoid obstacles. However, the overall transmission efficiency, the flight endurance, and the possible higher cost should be considered. Therefore, the multi-node mobile network integrating diverse flying platforms may be an approach to enhance the robustness and coverage of the network.

\section{System Design and Kernel Technologies}

Free-space QKD is now experiencing a comprehensive evolution towards a reconfigurable and integrated heterogeneous network exploiting multiple types of channels. Despite the numerous theoretical and experimental achievements for satellite ${ }^{[64]}$ and terrestrial free-space QKD mentioned above, airborne QKD is still in its infancy and leaves many practical issues to be solved. We will summarize and discuss the design methods and the critical techniques of the existing airborne QKD systems in this section.

\subsection{System composition}

Similar to the terrestrial free-space QKD systems, a typical airborne QKD system can be divided into the following modules according to their functions: quantum source, detector, optical calibration with the ATP, classical communication, and postprocessing. Compared with the ground-based free-space experiments, the most dramatic difference lies in the additional integration platforms and multi-stage ATP mechanisms. In ground-based QKD systems, some optical components or detection devices are deployed in detached positions to avoid background noise ${ }^{[65]}$, and there is usually no strict requirement for high-precision ATP systems to track and keep a stable optical link. Things become different in the airborne scenario since a limited payload is allowed for accommodating bulk QKD modules onboard. Besides, multi-stage high-precision ATP systems are indispensable to guarantee high and stable coupling 


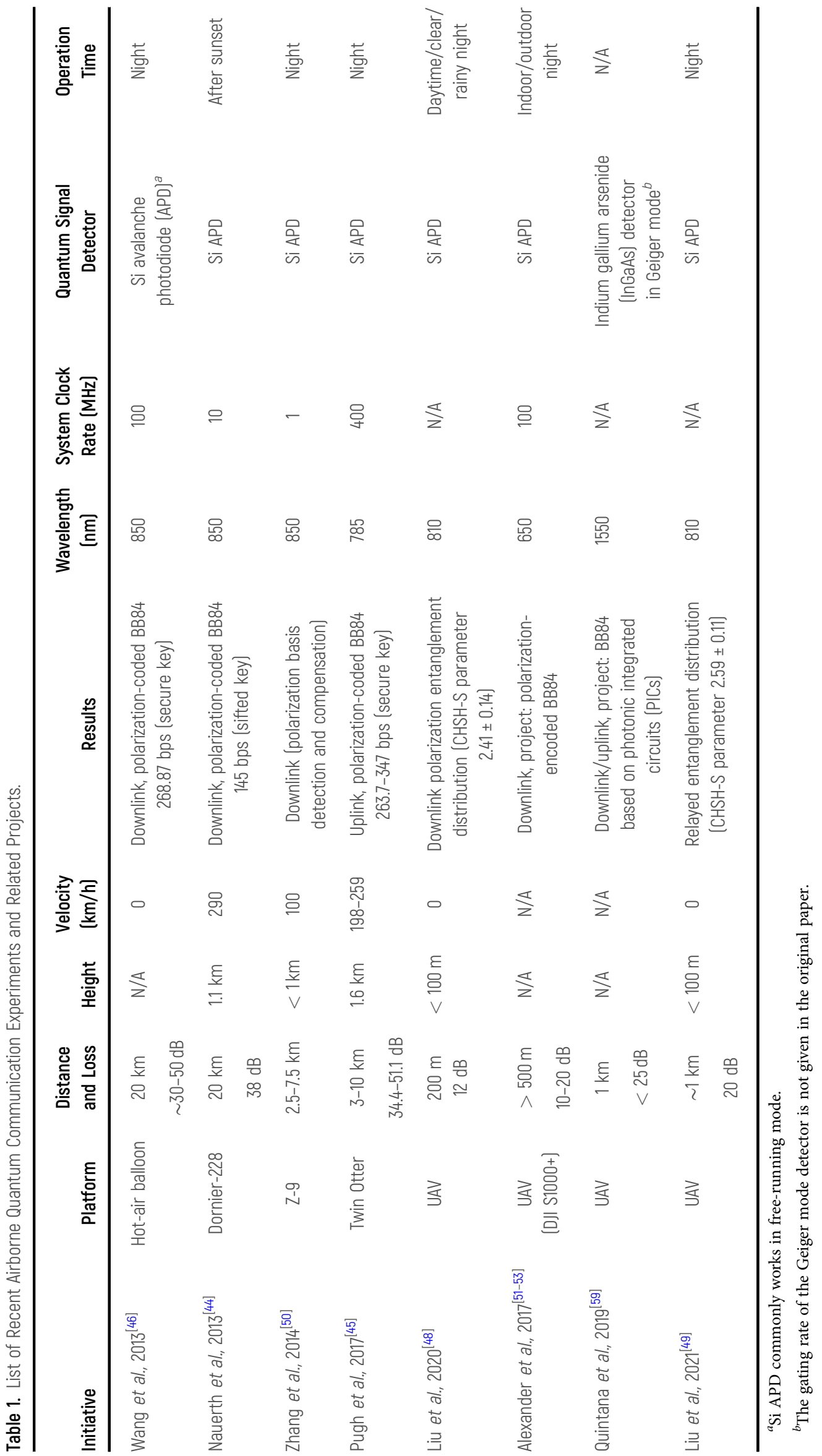


efficiency during the relative movements between the transmitter and receiver. Furthermore, a UAV platform may need more than one high-precision ATP system to satisfy multi-directional alignment demand.

Figure 3 shows the block diagrams of an airborne QKD system. The red dotted blocks present the quantum source and detector on each side. They are the core components for qubits preparation and measurement, which usually comprise the laser source, intensity, polarization or phase modulators, quantum random number generators, single-photon detectors, and other auxiliary devices for system compensation.

The imperfections of these devices may bring in side-channel loopholes and undermine the security of the practical QKD sessions. For the quantum source module, the parameters of the output signals like frequency, wavelength, line width, and intensity should be kept stable and consistent during the transmission procedure to avoid source-dependent attacks. For example, in an air-to-ground QKD experiment ${ }^{[44]}$, four laser diodes with nominal emission wavelength at $850 \mathrm{~nm}$ were used as the quantum source. Instead of using the single-mode fiber (SMF) as an optical filter, the authors developed a compact dummy laser diode package with a narrow spatial filter and collimation to eliminate the discrepancies among their wavelength distributions. It is worth noting that this method was easy to adopt onboard with limited power and space at the expense of the less optimal filtering effect. Auxiliary temperature and vibration control on the quantum source is still desired to guarantee more stringent indistinguishability of the laser signals.

The blue dotted blocks represent the optical calibration and ATP modules, which stabilize the optical link and maintain maximal coupling efficiency between remote terminals. The ATP modules are usually made up of coarse and fine pointing feedback loops according to the requirements of the specific applications. The coarse pointing loop aims at initial alignment for the beacon laser within the receiving field-of-view (FOV) and features in the wide scanning range and low bandwidth. The fine pointing loop is used for the accurate alignment and residual error correction after coarse pointing. As shown in Fig. 3, a position-sensitive detector (PSD), fast-steering mirror (FSM), and motor are typical apparatus to constitute a course pointing loop. ATP systems can be classified into the gimbalbased, mirror-based, gimbal-mirror hybrid, and others, which have been well investigated in mobile FSO communications ${ }^{[66]}$. The available execution and position sensor components of the coarse and fine pointing loops are diversified. Brushless direct current motors (BDCMs) and permanent magnet synchronous motors (PMSMs) are usually used to drive the coarse pointing telescopes. The piezoelectric FSM and voice coil mirror (VCM) are often used in fine pointing systems. The CCD camera, CMOS camera, or four-quadrant position detector (QPD) is essential for beam acquiring and detection in coarse and fine pointing loops.

The gray blocks denote the classical communication and postprocessing module, which have three major tasks. Firstly, this part will perform the basis reconciliation, error correction, and privacy amplification after the transmission of qubits is accomplished. Second, well-defined clock synchronization is also attributed to classical communication, which is beneficial to precise modulation and systematic coordination. Finally, all of the electronics used to support the QKD process and flying campaign are referred to as classical communications, including the time-to-digital converter, global positioning system (GPS) data transmission, command and control information distribution, etc.

The purple blocks indicate the hosting platforms that carry a transmitter or receiver. The way that they are connected to the QKD equipment is the major difference between the airborne QKD and other QKD systems. For the moment, there are three

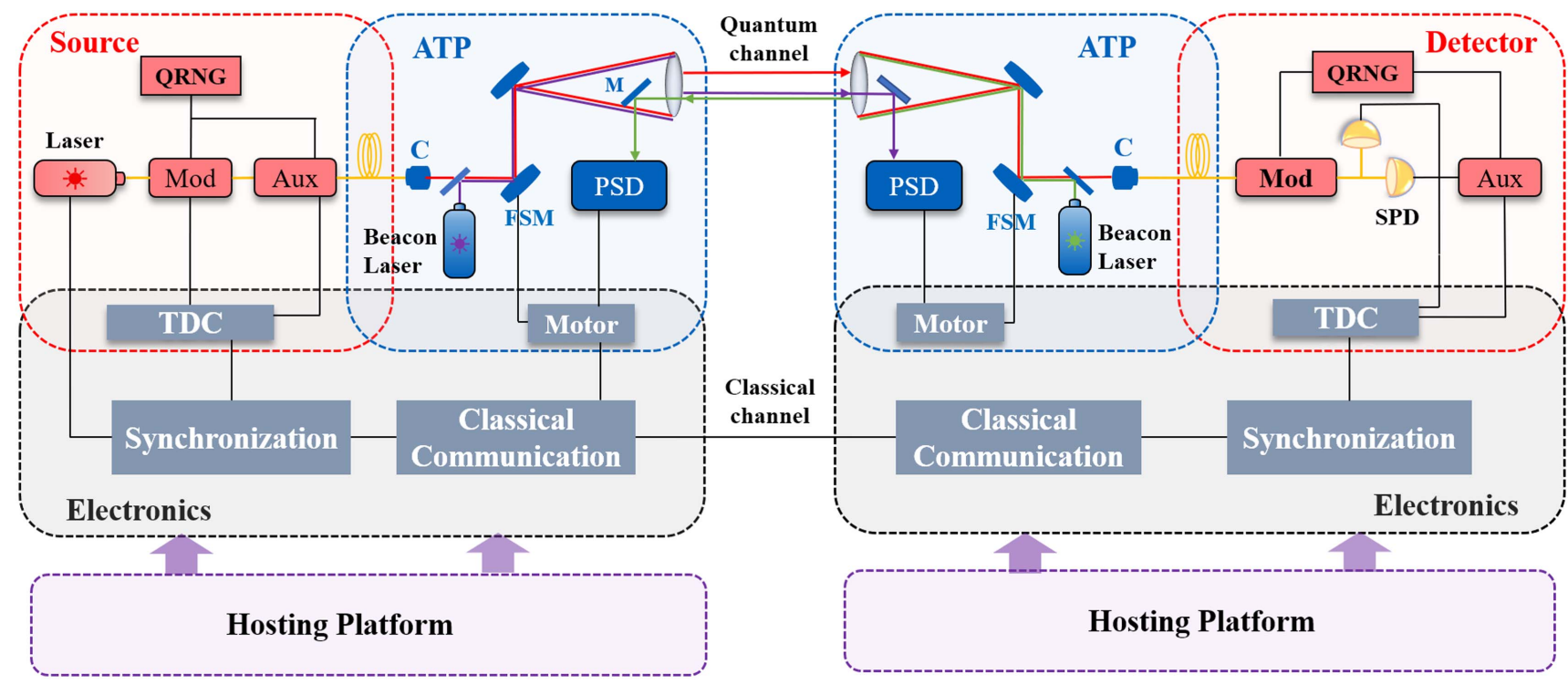

Fig. 3. Block diagrams of airborne QKD system. QRNG, quantum random number generator; Mod, modulator; Aux, auxiliary devices; TDC, time-to-digital converter; ATP, acquisition, tracking, and pointing; FSM, fast-steering mirror; PSD, position-sensitive detector; C, coupler; M, mirror; SPD, single-photon detector. 
ways to install and integrate the optical antennas onto the airborne platforms. First, using an external hanging pod to protect the optical devices is the most straightforward and cost-effective way to carry the QKD assembly. However, the system stability can be easily influenced by vibrations during take-off and landing due to the rigid coupling. What is more, this paradigm will change the aerodynamic characteristics of the airborne platforms to some extent and lead to strong local turbulences, which poses great challenges on signal receiving and detection. The second way is to equip QKD devices inside the cabin of the platforms. In this manner, one can fix the transmitter or receiver on the floor of an aircraft or helicopter and mount the telescopes used for the beam direction facing outside with the cabin door open or removed. This approach is beneficial for real-time maintenance during flight and carries a heavy payload at the same time. Nonetheless, it has an obvious drawback in that the angular motion range of the telescope is limited due to the width of the cabin door ${ }^{[45]}$. The third manner is to integrate the QKD components with the fuselage. In other words, the optical terminal contains part of the aircraft itself. It has been demonstrated in the air-to-ground QKD experiment, where the optical antenna was covered by a hemispherical hood beneath the Dornier-228 utility aircraft, as shown in Fig. 2. This approach is advantageous to provide a proper shield from stray light and good stability during flight. It has little impact on the aircraft's aerodynamic status but lacks in-operation flexibility.

\subsection{Link configurations and the channel loss}

Concerning different scenarios that allow the transmission of qubits, the airborne platforms can play the role of a transmitter, receiver, or even an optical relay node. Figure 4 illustrates three possible cases for airborne quantum communications. In the uplink configuration of Fig. 4(a), the quantum transmitter is either placed at the ground station or portable station, where line-of-sight links can be established with a moving aircraft ${ }^{[67]}$. The advantage of the uplink configuration is that the quantum source can be designed elaborately and deployed with adequate protections. Moreover, the aircraft can also send quantum signals downward to ground-based receivers, which is noted as the downlink configuration, as shown in Figure 4(b). In this configuration, the receiving side benefits from using the largeaperture telescopes to enhance the beam collection efficiency. Sophisticated and relatively bulky superconducting nanowire single-photon detectors (SNSPDs) ${ }^{[68,69]}$ can also be utilized to improve the detecting efficiency of quantum signals. By utilizing the drones with a high-precision ATP system, it is also feasible to realize flexible inter-platform links, as shown in Fig. 4(c), which means that it is promising in supporting separate users within a local area that satellites cannot access in time by building a scalable airborne network.

In satellite $\mathrm{QKD}$, the loss characteristics of the link and the receiver can be summarized as atmosphere attenuation, space coupling loss to the receiver telescope, the receiver's telescope loss, and quantum receiver loss ${ }^{[70]}$. The atmosphere attenuation, which is the most variable and uncontrollable, mainly suffers from absorption, scattering, and turbulence. These effects will lead to intensity fluctuation, beam spread, spot quiver, and pathlength fluctuations to the laser beam and finally affect the signal to noise ratio (SNR), clocking synchronization, and the QKD system.

The atmospheric turbulence is an intractable barrier causing beam distortion and transmission efficiency fluctuation in freespace quantum channels, especially in the near-surface channel. Fortunately, the turbulence intensity characterized by the refractive index decreases with the smaller zenith angles ${ }^{[71]}$, and the airborne platforms can move to search the positions with small zenith angles and lower background noise to get better channel transmittance and SNR. Bourgoin et al. have made a comprehensive analysis on the free-space transmission statistics and the QKD performance in different links ${ }^{[72]}$. They proved that the key generation rate of an uplink configuration is roughly one magnitude lower than that of the downlink, which is mainly due to the asymmetric distribution of the atmospheric turbulence in a vertical channel.

Therefore, stratospheric platforms are slightly subjected to unstable airflow and turbulence. Moll et al. have analyzed the

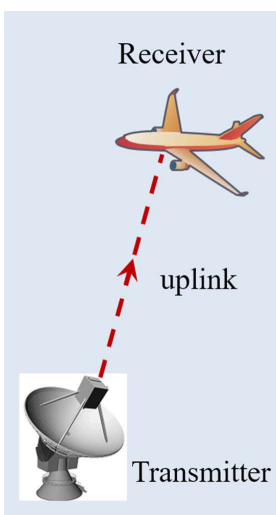

(a)

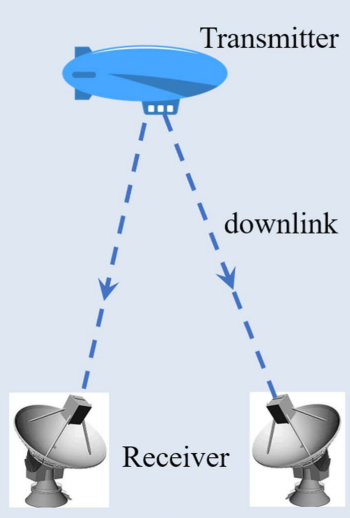

(b)

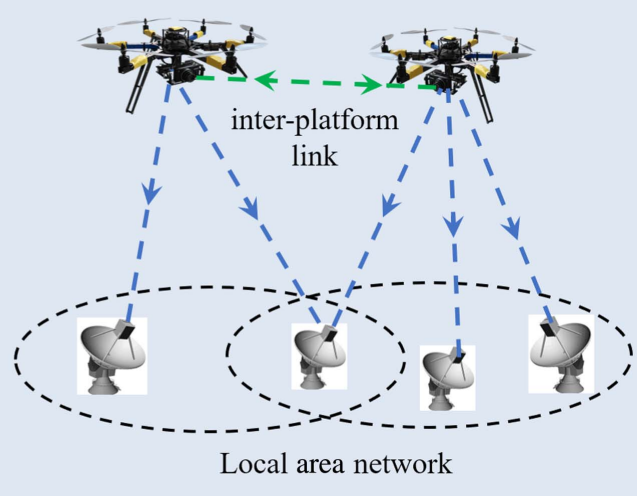

(c)

Fig. 4. Link configurations for airborne QKD. 
feasibility and preferability of implementing both discretevariable $\mathrm{QKD}$ (DV-QKD) and continuous-variable $\mathrm{QKD}$ $(\mathrm{CV}-\mathrm{QKD})$ in the stratosphere ${ }^{[73]}$. Since the turbulence is more concentrated near the surface and does not depend on the transmitter's aperture, the turbulence-induced beam wandering and broadening will worsen for an uplink path, where the laser beam encounters turbulence at the very beginning of the free-space channel.

Another important factor leading to the transmission loss of quantum communication in free space is the diffractioninduced beam broadening, which is determined by various factors such as the wavelength of the laser, the propagation distance, and the apertures of the transmitter and receiver. This is also known as geometric loss, a core parameter in evaluating the performance of QKD. In general, larger receiving apertures or smaller transmitting divergences result in less geometric loss for a given range according to the formula ${ }^{[74]}$

$$
\eta_{\text {diff }}=10 \log \left(\frac{D_{r}}{D_{t}+\theta L}\right)
$$

where $D_{r}$ and $D_{t}$ are the apertures of receiving and transmitting devices, and $\theta$ and $L$ are the divergence angle and link distance, respectively. However, with the addition of atmospheric turbulence, this diffraction loss could be more significant in the uplink case than in the downlink one ${ }^{[75]}$ :

$$
\eta_{\text {diff }}^{\text {up }}=\frac{L^{2}\left[\left(\frac{\lambda}{D_{t}}\right)^{2}+\theta_{\text {turb }}\right]}{D_{r}^{2}} \frac{1}{T_{t} T_{r}\left(1-L_{p}\right)} 10^{A_{\text {atm }} / 10}
$$

where $\theta_{\text {turb }}$ is the beam divergence caused by atmospheric turbulence. $\lambda$ is the wavelength of the beam. $T_{t}, T_{r}, L_{p}$, and $A_{\text {atm }}$ are the transmission factor, receiving factor, pointing loss, and atmospheric attenuation, respectively. Using the equations above, we could roughly derive the link budgets for different scenarios. Assume that the apertures of the transmitter and receiver are $26.4 \mathrm{~mm}^{[48]}$, the beam wavelength is $1550 \mathrm{~nm}$, and the divergence angle is within the diffraction limit. The transmission and receiving factors are 0.5 with no pointing loss. For a $1 \mathrm{~km}$ uplink, the channel loss is nearly $7 \mathrm{~dB}$, while the downlink is about $5 \mathrm{~dB}$.

The asymmetric channel loss of different configurations with the same altitude can significantly differ in a satellite-based QKD system design and practical operation. Whereas airborne platforms fly at a much lower altitude than the LEO satellites, the total link budget has minor deviations between the uplink and downlink in terms of diffraction loss, atmospheric turbulence, and other types of attenuation. What should be noted is that one can achieve low diffraction loss within a long-distance path by dividing it into several short segments and exploiting the optical relay method ${ }^{[49]}$, which is a feasible way for drone-based platforms. In the first two cases, both the ground stations and the airborne platforms are regarded as trusted users. Otherwise, the flying platforms are functioning as untrusted nodes linking two remote ground stations in case the dedicated fiber channel is spoiled. The third case could be helpful in securing the wireless communications between two aircraft or between the satellite and aircraft with an ad hoc networking manner ${ }^{[76,77]}$ in analogy with the classical wireless network. It can serve as an intermediate link connecting the spaceborne nodes and terrestrial quantum networks. Although the QKD experiment demonstrating the third link configuration is still missing for the moment, its potential in wide applications is predictable once the highaccuracy self-adaptive ATP techniques and compact QKD systems are mature enough.

\subsection{Generating and manipulating high-quality quantum optical signals}

Optical technologies are the most mature approaches to implement quantum communications, and the performance of the QKD system significantly depends on the quality of the light source. The ideal single-photon source that contains only one photon per pulse is the best security solution. Unfortunately, the ideal single-photon source is far from practically available, even if a few photons can be generated by quantum dots ${ }^{[78]}$ or spontaneous parametric down-conversion (SPDC) methods ${ }^{[79]}$. Alternatively, the weak coherent pulse (WCP) with phase randomization $^{[80,81]}$ is a typical solution by virtue of its simplicity and good performance, and the decoy-state method helps the practical WCP to beat the photon number splitting (PNS) attack $^{[23,24]}$. In addition, the quantum light source should be with light weight and easy to integrate with existing FSO communication instruments for the airborne QKD.

Polarization encoding is the preferred scheme to implement free-space QKD. For a polarization-encoded system, the polarization extinction ratio (PER) is the primary parameter to characterize the quality of the quantum signals and determines the intrinsic errors of the QKD system.

Figure 5 shows a comparison of the simulated key rate of a decoy-state polarization-encoded QKD system with different values of PER.

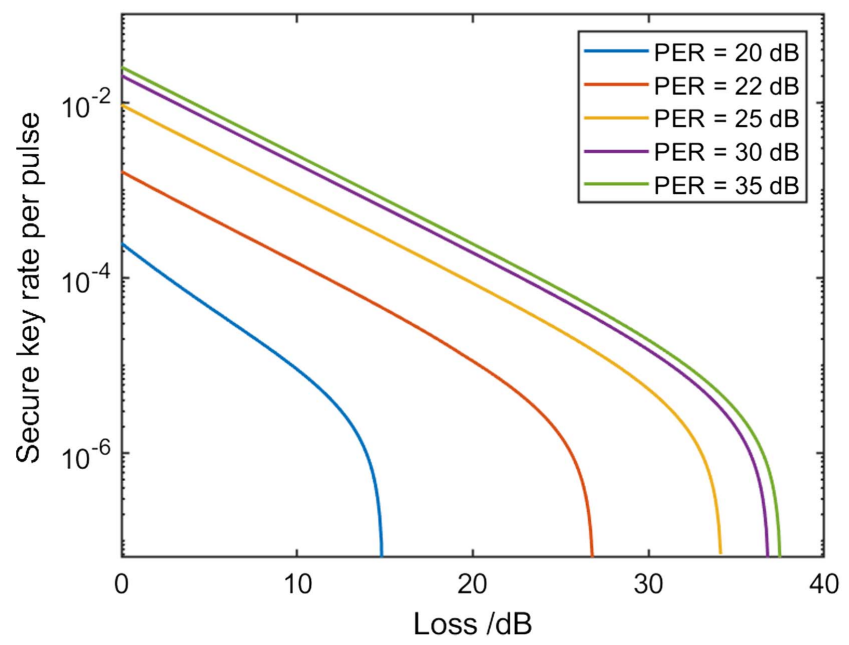

Fig. 5. Secure key rates with different PER. 

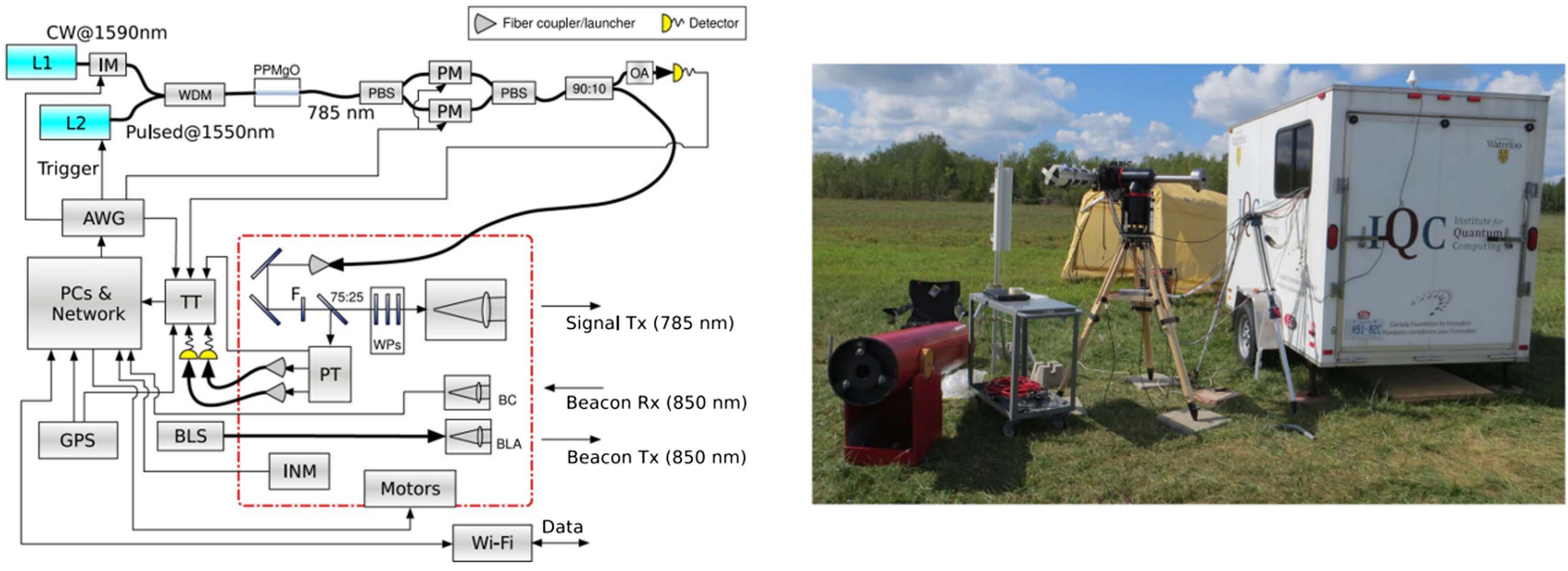

Fig. 6. Quantum source and transmitter in the ground-to-air QKD demonstration ${ }^{[45]}$

It is suggested that the PER of the transmitting signal in a freespace polarization-encoded QKD system should be higher than $20 \mathrm{~dB}^{[82]}$. In 2007, Peng et al. used eight distributed feedback (DFB) laser diodes to generate the high PER signal and decoy states, respectively ${ }^{[83]}$. This scheme is inherited into their follow-up satellite-to-ground QKD experiment, where the frequency is enhanced from $2.5 \mathrm{MHz}$ to $100 \mathrm{MHz}^{[41]}$.

In 2013, two representative airborne QKD experiments were reported simultaneously. The two groups from China and Germany both employed the four-laser diode quantum source scheme. The light source in Ref. [46] was composed of four laser diodes working at $850 \mathrm{~nm}$. The two pairs of orthogonal states $|H\rangle,|V\rangle,|+\rangle,|-\rangle$ are modulated by two polarization beam splitters (PBSs), one beam splitter (BS), and one half-wave plate (HWP). Under the control of a high-speed random number generator, signals of different types and amplitudes were emitted at a repetition frequency of $100 \mathrm{MHz}$. In order to improve the PER of the light source, all the mirrors are coated with metal films besides using custom-made high extinction ratio optical elements (above 1000:1).

In Ref. [44], they used four laser diodes, which are intrinsically linearly polarized (typically PER of 1000:1, i.e., $30 \mathrm{~dB}$ ). Then, the emitted pulses encoded with four polarization states are driven by a $100 \mathrm{MHz}$ clocking field programmable gate array (FPGA) that generates short pulses with $10 \mathrm{MHz}$ repetition rate and $1 \mathrm{~ns}$ pulse width. It is intuitive and convenient to modulate multiple lasers separately or side-by-side. In this way, the SMF coupling method is indispensable for obtaining both spatial consistency and coaxiality. However, coupling to the SMF requires refined temperature stabilization and anti-vibration measures due to the polarization mode dispersion (PMD) effect. Wang et al. added a monitoring power meter to ensure that the pulse amplitude is consistent. In contrast, Nauerth $e$ t al. added narrow-band spatial filtering and a servo arm equipped with attenuator diodes to calibrate the pulse intensity in real-time.

It should be noted that if the multi-laser scheme is adopted, the parameters of photons from different lasers, such as the wavelength, repetition frequency, pulse width, PER, etc., should be kept indistinguishable to eliminate the risk of the sidechannel loopholes ${ }^{[84,85]}$. Therefore, the scheme with a single laser is a better solution from the viewpoint of practical security.

In 2010, Jofre et al. proposed a free-space polarizationencoded QKD experiment using a single laser with a frequency of $100 \mathrm{MHz}^{[86]}$. In 2013, Yan et al. developed a single-laser scheme based on the sum-frequency generation (SFG) process to generate a polarization-stabilized pulsed laser sequence, which was successfully modified and applied in the later airborne experiments ${ }^{[87]}$.

Using a single laser with multiple optoelectronic modulators is also an available solution for airborne QKD. From 2015 to 2017, the group led by Jennewein has conducted a series of QKD experiments based on mobile platforms, including truck and aircraft ${ }^{[45,54]}$. In those experiments, a continuous-wave laser at $1590 \mathrm{~nm}$ and a pulsed laser at $1550 \mathrm{~nm}$ were combined to generate a quantum source at $785 \mathrm{~nm}$ through SFG. Using this nonlinear sum frequency conversion, the quantum light source has the benefit of both a short coherent length of a $1590 \mathrm{~nm}$ laser and the same pulse rate as the mode-locked laser. Therefore, it is possible to incorporate an advanced and flexible light source with high-speed modulation to obtain better environmental stability. The QKD source and part of the electronics were located inside of a trailer to maintain the temperature and humidity stability in their experiments, as shown in Fig. 6.

An intensity modulator was used to modulate the photons to the signal states, decoy states, and vacuum states, respectively. A balanced Mach-Zehnder interferometer (MZI) with a phase modulator was used to modulate the photons to the horizontal, vertical, diagonal, and anti-diagonal polarization states.

\subsection{High-precision ATP and polarization compensation}

ATP is a kernel component to establish FSO links and maintain alignment between the transmitter and receiver. It has upgraded from two-dimensional gimbal-stage mechanisms to adaptive optics and liquid-crystal-based systems in the FSO system and has been reviewed comprehensively ${ }^{[66]}$. Different types of 
ATP systems have various applications as well as pointing performance. Nonetheless, there are two special considerations when designing the ATP system for free-space quantum communications. Firstly, in FSO communications, the pointing and coupling loss can be compensated by increasing the transmitting power.

However, this is infeasible in quantum scenarios for security reasons, such as the no-cloning theorem of single photons. Reducing the background noise and keeping a steady highefficiency optical alignment by using the ATP is a possible way to enhance the SNR. Secondly, polarization deflections are inevitable during long-distance free-space propagation with dramatic relative motions between two sides. Besides, the massive mirrors and coatings used in the ATP systems will introduce unnecessary birefringence to the quantum states ${ }^{[88,89]}$. To this end, the polarization compensation ability and the high accuracy of the ATP system should be considered simultaneously.

For satellite-based QKD, the ATP system and its controlling algorithm can be designed according to the orbiting routine and flying data. For example, in the in-orbit test of the optical link of the satellite-to-ground QKD experiment ${ }^{[90]}$, the ground station calculates first the pointing angle in line with the heralded orbit information and leads the wide-divergence beacon light to the satellites' uncertain region. Then, the satellite will adjust its altitude and point at the ground station with the accuracy of about $0.5^{\circ}$ following the predicted guidance. Multiple-stage ATP systems were applied both in the transmitter and receiver. The divergence angle of the beacon laser on each side is $1.25 \mathrm{mrad}$ and $0.9 \mathrm{mrad}$, respectively. The angles are larger than the tracking accuracy of $1.2 \mu \mathrm{rad}$ based on the closed loop feedback algorithm. However, for low-altitude flying platforms, the short link and high velocity mean faster angular speed, which necessitates the ATP system with higher precision and more efficient feedback and control algorithms.

The high-precision ATP system for quantum communication often adopts the gimbal mirror hybrid structure to perform the bidirectional tracking and pointing. Generally, two sets of control loops to perform the coarse and fine pointing sessions are contained on each end, and their tracking errors are typically about several hundred and several microradians, respectively.

The quantum beam's divergence angle is commonly narrowed to enhance the link efficiency. The typical divergence value is approximately $180 \mu \mathrm{rad}$ in the air-to-ground $\mathrm{QKD}$ experiment $^{[44]}$ and $500 \mu \mathrm{rad}$ in the hot-air balloon platform ${ }^{[46]}$. Besides, two-stage ATP systems are commonly adopted on the airborne platform and the corresponding ground station. The coarse pointing loop used the InGaAs wide FOV camera (48 mrad) and motorized Kepler telescope on the Dornier228 aircraft $^{[44]}$. Fine and fast corrections to the coarse pointing were enabled by the VCM, digital signal processor (DSP), and four-quadrant diodes (4QDs). The coarse pointing loop at the ground station was composed of the InGaAs camera with 12.8 FOV and torque motor-driven telescope. The fine pointing was realized by a piezo actuated mirror and narrow FOV InGaAs camera (960 $\mu \mathrm{rad})$. The ATP performance parameters in recent airborne demonstrations are summarized in Table 2.
According to the existing studies, the divergence angle and tracking accuracy for future experiments should be aimed at the magnitude of less than $10 \mathrm{mrad}$ and higher than $5 \mu \mathrm{rad}$, respectively.

Before the ground-to-air QKD demonstration, Bourgoin et al. ${ }^{[72]}$ derived an advised tracking error of $2 \mu \mathrm{rad}$ at the ground-based transmitter and $20 \mu \mathrm{rad}$ at the airborne receiver. At each side of the transmitter and receiver, a beacon laser assembly (BLA) made up of three fiber launchers with fixed divergence $(\sim 13 \mathrm{mrad})$ and a two-axis motorized telescope is installed to provide coarse pointing. Moreover, inertial navigation modules (INMs) containing GPS receivers and altitude sensors are also exploited for coarse pointing. The two sides exchange their own GPS data in real-time via a classical wireless fidelity (Wi-Fi) link, which is not possible in a satellite-based scheme.

As for the drone-based entanglement distribution demonstration by Liu et al. ${ }^{[48]}$, strong wind and the motion of propellers aggravate the swing of the drone and put higher requirements on the ATP systems. They designed and customized a pair of ATP units to achieve bidirectional pointing, as shown in Fig. 7. On each side, PSD is used to detect the beam spot centroid and send feedback signals to control the FSM for fine pointing. The threeaxis gimbal motorized telescope and coaxial zoom camera are coordinated to perform coarse pointing in a similar way.

The detection results are only significant to generate secure key bits when the transmitter and the receiver select the same bases. For example, in a polarization-encoded QKD system, the overall polarization degradation is mainly derived from the base deviations due to the relative motions of the transmitter and the receiver, which will make the so-called shared reference frames inconsistent. Unlike the stationary systems, in the mobile or airborne scenario, the high-fidelity transmission of the quantum states and the reference frame calibration are not trivial tasks to be accomplished or even critical technical challenges.

There are several schemes to improve the polarization fidelity of the quantum states of the transmitted photons. The first one is to optimize the design of the optical module either by minimizing the incident angle or making the mirrors and wave plate deposited in orthogonal positions to maximize the polarization visibility. This method was fully verified in the air-toground QKD demonstration ${ }^{[44]}$. The second one is to adopt the reference-frame-independent protocols ${ }^{[91,92]}$ to immunize the system from slow rotations of polarization bases. Zhang et al. proposed to make real-time compensation on the drifting bases according to the ephemeris and anticipated orbiting data ${ }^{[50]}$. They also developed a basis-deviation detection scheme and a compensation approach based on single-photon detection to optimize the ATP's performance. However, the referenceframe-independent (RFI) protocol requires enough accumulation of raw data to conduct parameter estimations, which is still hard to achieve in satellite-based QKD when considering the finite-size effects. This dilemma could be properly alleviated for the airborne QKD scenarios taking advantage of the increase of the transmission efficiency. 
Table 2. ATP Performance of the Typical Airborne Quantum Communication Experiments.

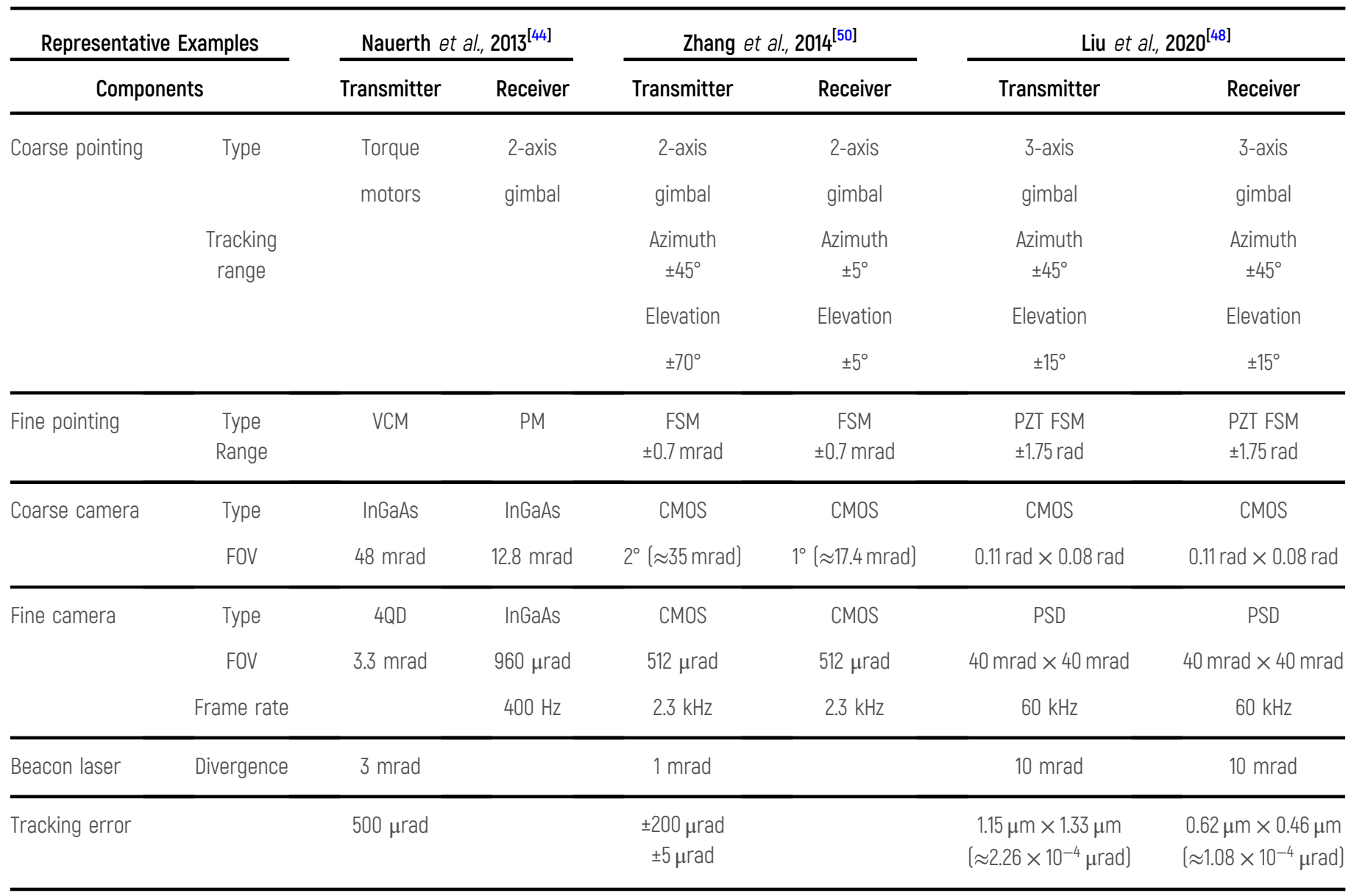

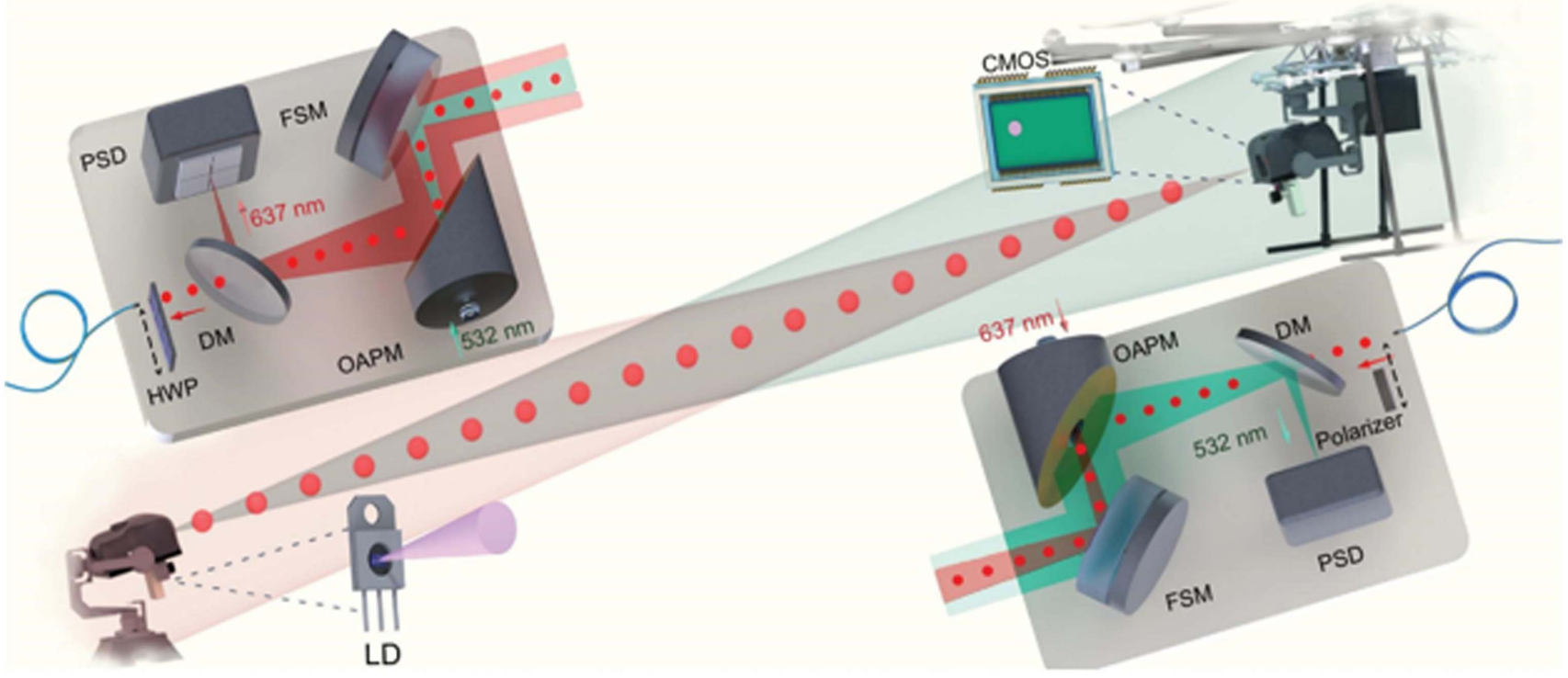

Fig. 7. ATP system in the drone-based entanglement distribution experiment ${ }^{[48]}$. 
The polarization compensation is an intuitive method adopted in airborne quantum communications. Pugh et al. designed a correcting system comprising a polarization tomography and motorized wave plate assembly at the transmitter ${ }^{[45]}$. Nauerth et al. attempted the close loop and open loop control schemes to manage the tardy polarization rotations ${ }^{[44]}$. The close loop control was to determine the angular positions of the waveplates by measuring the polarization rotation of the bright calibration signals over the quantum channel, which are usually two non-orthogonal states. The calibration signals, which are sent occasionally, and the duty cycle depend on the trajectory of the airplane. Although the scheme is able to completely characterize the birefringence of the quantum channel, in principle, it is difficult to obtain reliable measurements with a practical polarimeter in a short interval, and the duty cycle may vary due to the fluctuations of the channel.

The second manner was to carry out an open loop polarization compensation procedure based on carefully characterizing relevant system components, such as the static birefringence of the optics device in the aircraft and the ground station, the phase shifts introduced by the mirror with different light incident angle or the optical dome the light passed, and the ration around the beam axis introduced by the flight altitude of the aircraft. All of these decomposed distortions to the polarization can be either measured in advance or calculated from the live data of the flight terminal and the station. Therefore, the open loop method can be, in principle, performed without the auxiliary calibration light and additional intervals. Therefore, the open loop scheme is able to keep the quantum transmission uninterrupted, which is advantageous, especially in view of the finite-size effects. It is foreseeable that the practical effects of the open loop method will be related to the algorithm and the correctness of the pre-determined system parameters.

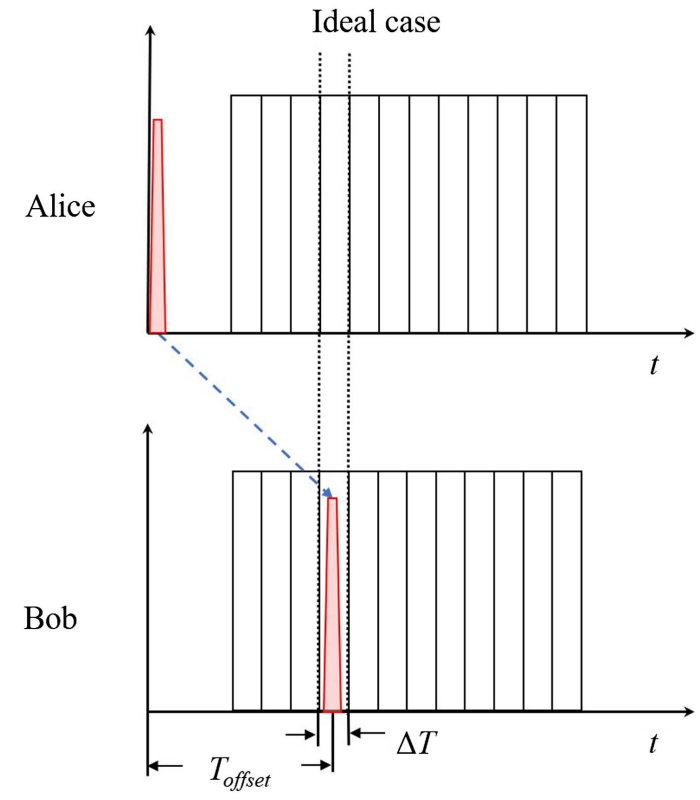

\subsection{High-precision synchronization}

Precise synchronization is indispensable for a QKD system for at least two reasons ${ }^{[93]}$. First, it serves as a time gate filter to discriminate between the quantum signals and the background noise introduced either by the detector or the eavesdropper. Second, unlike classical communications, signals at the singlephoton level are hard to encode in a complex temporal mode. Therefore, the sync signal is necessary to precisely correlate between the transmitted qubit sequence of Alice and the detection events of Bob.

The synchronization methods such as electrical-signal synchronization ${ }^{[94,95]}$, atomic clock synchronization ${ }^{[96]}$, and optical synchronization ${ }^{[97]}$ have been adopted in some experiments, and synchronizations using GPS, ultra-stable crystal oscillator, and self-entanglement are also utilized ${ }^{[39,98,99]}$. However, for free-space quantum communication, especially for QKD based on fast-moving platforms, additional beacon light is often used to facilitate ATP and system synchronization simultaneously. Thereby, it has been demonstrated in several cases that optical synchronization is a feasible solution that can simplify the burden of the system and provide a high-precision time benchmark.

As shown in Fig. 8, there is a fixed time offset $T_{\text {offset }}$ between the receiving qubit and the sending qubit ideally. By adding a tunable delay module to the receiver side, the pulses of each side could be calibrated. Nevertheless, the time offset in practice is often variable due to factors such as the light source instability, transmission fluctuation, and timing jitter of the detectors, which commonly leads to the Gaussian distribution of the received quantum signals.

If the signal temporal expansion is too wide, the system cannot distinguish whether the incident light pulse is a dark count or a quantum signal within the coincidence window. Assume that the quantum bit error rate (QBER) can be attributed to

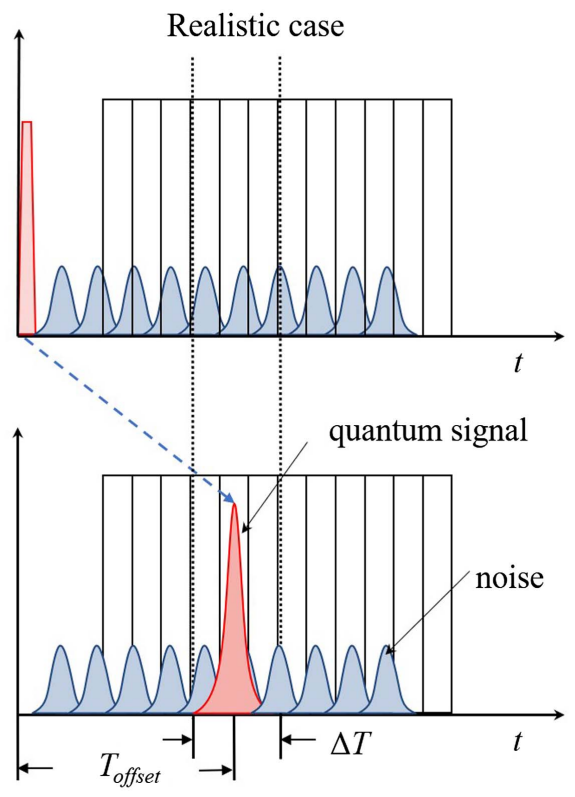

Fig. 8. Schematic diagrams of time synchronization precision. 
imperfections, including the source error $e_{s}$, transmission error $e_{t}$, measurement error $e_{m}$, and the error caused by imprecise synchronization. The total error is expressed as ${ }^{[100]}$

$$
e=e_{s}+e_{m}+e_{t}+\frac{P_{\text {noise }}}{2\left(P_{\text {signal }}+P_{\text {noise }}\right)},
$$

where $P_{\text {noise }}$ is the probability of noise counts per unit time, and $P_{\text {signal }}$ is the signal responding probability per unit time. Considering the synchronization accuracy, $P_{\text {noise }}$ can be calculated as the product of noise count rate $N$ and coincidence window $\Delta T$. It implies that improving the system synchronization accuracy is conducive to reduce the coincidence window and improve the SNR. Wang et al. ${ }^{[46]}$ adopted a hybrid method of GPS synchronization and optical beacon synchronization, which reached an accuracy of $1 \mathrm{~ns}$. In the air-to-ground experiment, the classical optical signal is used for synchronization. For Liu et al.'s UAV-based quantum entanglement distribution experiment ${ }^{[48]}$, the electrical signals of the detector are converted to an optical signal through a $300 \mathrm{~m}$ fiber for synchronization to synchronize the coincidence counts of the two parties.

\section{Challenges and Perspectives}

Free-space QKD is an attractive topic and is still in its beginning stage, which is far from wide-scale applications. However, it is foreseeable to be broadly applied soon, especially for military fields like secure communication, accurate navigation, and precise situational awareness ${ }^{[101,102]}$. Airborne QKD can provide flexible quantum security for military clients who cannot easily refresh their secret keys.

Realizing real-time, all-weather, and global coverage QKD is the primary stage to build the quantum internet. In the next step, dividing long-range quantum links into many segments through quantum entangle distribution and quantum memory gradually comes into being. Airborne QKD will function as effective relaying nodes by getting rid of spatial and temporal constraints. In general, airborne quantum communication is not only a powerful measure to protect the existing wireless communication, but also an important means to accelerate the integration of quantum information technology with other technologies. At present, airborne quantum communication was only experimentally verified by a few groups. For fixed-wing aircrafts, the flying tests are mainly with the line or circular routines. For drone-based demonstration, the platform hovers in the air and did not perform complicated maneuvers or rapid movements. The technology readiness level (TRL) of airborne quantum communication is still in the low stages. It is necessary to improve several of the following aspects to make this technology more applicable in real life.

\subsection{Precise, rapid, and adaptive ATP}

Despite numerous researches of ATP in classical communications, the requirements of the small divergence angle, random relative motion, and high SNR in airborne quantum communication still pose technical challenges to improve its accuracy. As mentioned above, predetermined straight-line or arc-line trajectories or hovering is beneficial to reduce the initial capture time and continuous tracking of the mobile terminals. The gimbal-stage mirror or FSM controlled by the fast-executing proportion integration differentiation (PID) algorithms is qualified to reach high accuracy and low overhead.

However, in practical applications, the flight path is not always fixed. Natural objects may occasionally block the lineof-sight due to the flying path variations, which will interrupt the beam coupling. Consequently, ATP combined with adaptive optics is suggested to be used together with intelligent feedback algorithms to search the target automatically and establish the alignment accurately. Second, the varying wavefront distortion caused by the turbulent atmosphere should be suppressed when designing ATP. Moreover, the trade-off between the apertures of the pointing telescope and its angular resolution should be reflected according to the basic diffraction limitation ${ }^{[101]}$ :

$$
\Delta \theta=1.22 \frac{\lambda}{D} \mathrm{rad},
$$

where $D$ is the diameter of the telescope aperture, and $\lambda$ is the wavelength of the transmitting beam.

Another critical technical challenge of airborne quantum communication is the random altitude change of the platform such as roll, pitch, and yaw, which increases the difficulty of optical signal receiving and detecting and puts forward higher requirements on ATP systems. According to the airborne QKD demonstrated experimentally by Pugh et al. ${ }^{[45]}$, the maximum angular speed was about $0.5^{\circ}$ to $0.7 \mathrm{~s}^{-1}$ during the beacon pointing lock. The QBER varies from $2.96 \%$ to $5.24 \%$ with the locked link, and the channel loss varies from $34.4 \mathrm{~dB}$ to $51.1 \mathrm{~dB}$. These results vary dramatically and are often higher than theoretical simulation results due to varying pointing accuracy ${ }^{[45]}$. It can be predicted that the high-frequency vibration and low-frequency jitter of the airborne platform will enlarge the alignment error of the transmitted beam and reduce the receiving power, especially as the distance increases. Therefore, anti-vibration and self-stabilized devices to implement adaptive ATP with faster response time and higher accuracy are indispensable in airborne quantum experiments.

\subsection{Miniaturization of the airborne devices}

The power and space consumptions may be trivial to terrestrial experiments but vital to the airborne platforms, especially for UAVs or drones with limited payload. It is usually quoted as the size, weight, and power (SWaP) constraints ${ }^{[103]}$. This problem has been investigated in a lot in satellite-based QKD schemes. For example, the spaceborne payload on the Micius satellite is $132.46 \mathrm{~kg}$, including the quantum transmitter, ATP system, and general control box. The payload was reduced to $57.9 \mathrm{~kg}$ in the Tiangong-2 space QKD mission ${ }^{[104]}$ instead, 
including only the QKD transmitter and the ATP system. This weight seems too heavy for airborne platforms, especially UAVs. In fact, a commonly used DJI Phantom 4 picture drone has only a take-off weight of $1.4 \mathrm{~kg}$. With customized octocopters, this value can only reach $35 \mathrm{~kg}^{[48]}$, which means that the future QKD devices should be limited to tens of kilograms for the sake of airborne integrity.

As mentioned above, increasing the transmitting and receiving apertures is an efficient way of reducing the diffraction loss in free-space QKD. However, large optical devices burden the airborne platforms with limited SWaP ability. Besides, siliconbased single-photon detectors and InGaAs-based detectors exhibit different performances in airborne quantum communications. The former has a wide responding spectrum that provides flexible wavelength choice for the signal laser, and the latter is suitable for infrared photons detection but has relatively low detecting efficiency. They are both preferable to the bulk superconducting detectors, considering the payload limit.

Generally, the performance of QKD relates to the SWaP capacity in practical scenarios. Therefore, miniaturization of the QKD systems based on small-sized or tiny components should be considered in the next step. Some groups have been devoted to developing handheld ${ }^{[105]}$ and compact polarization analyzers for mobile free-space QKD. Fortunately, the implementation of QKD on photonic chips has developed rapidly in recent years ${ }^{[106,107]}$. Predictably, the chip-based QKD devices ${ }^{[108]}$ will significantly facilitate the development of airborne quantum communications by taking advantage of their excellent scalability, high stability, and repeatability.

\subsection{Mitigating the influence of aero-optical effects}

In satellite-based QKD sessions, most of the beam propagation happens in outer space. However, the airborne platforms are constantly operating within the atmosphere where the influence of turbulence cannot be overlooked. The effects such as beam wander, beam broadening, and scintillation caused by turbulence are detrimental to the process of signal tracking and receiving. It is noteworthy that the interaction between the airflow and the fuselage or propeller is a significant turbulence source, with either fixed-wing aircraft or rotary-wing UAVs. In some extreme cases, this interaction can even form a boundary layer due to the viscosity effects of the air, which will cause drastic fluctuations of the local refractive index and coupling efficiency of the QKD systems ${ }^{[109,110]}$. It is an interesting and worthwhile work to reduce or avoid these impacts. For future real-life airborne quantum communications, the optical antenna, adaptive SMF coupling method, and reasonable aerodynamic designs should be incorporated to enhance the system performance further.

Finally, in addition to technical revolutions, a mature airborne QKD system should develop the theoretical models and parameters optimizations to get the best performance by considering large statistical fluctuations due to the versatile characteristics of the airborne channel and the system itself.

\section{Conclusion}

The architecture of reconfigurable and long-distance hybrid QKD schemes represents a key strategy to the success of a full-fledged quantum network. At present, a number of research works on airborne quantum communications have been reported. The platforms have also been successfully exploited in some special applications, such as the verification of the satellite-based QKD.

The future airborne platforms will be enhanced with more sophisticated flying control techniques and finer optical wavefront reshaping methods. As the competitive edges of airborne quantum communication, the scalability and flexibility are destined to be upgraded from $100 \mathrm{~m}$ to nearly $20 \mathrm{~km}$ and from point-to-point links to complicated multi-node networks.

It is foreseeable that airborne quantum communications will play a fundamental role in the global secure quantum network, whether being exploited as an individual or transitional communication approach. Therefore, the research of airborne quantum communications remains a blue ocean, showing great opportunities to explore the boundaries of quantum mechanics.

\section{Acknowledgement}

This work was supported by the National Key Research and Development Program of China (No. 2018YFA0306400), National Natural Science Foundation of China (Nos. 61627820, 61822115, 61971436, and 61675189), and Anhui Initiative in Quantum Information Technologies (No. AHY030000).

${ }^{\dagger}$ The authors contributed equally to the work.

\section{References}

1. C. H. Bennett and D. P. DiVincenzo, "Quantum information and computation," Nature 404, 247 (2000).

2. A. Galindo and M. A. Martin-Delgado, "Information and computation: classical and quantum aspects," Rev. Mod. Phys. 74, 347 (2002).

3. C. H. Bennett and G. Brassard, "Quantum cryptography: public key distribution and coin tossing," in Proceedings of IEEE International Conference on Computers, Systems, and Signal Processing (IEEE, 1984), p. 175.

4. A. Ekert, "Quantum cryptography based on Bell's theorem," Phys. Rev. Lett. 67, 661 (1991).

5. V. Scarani, H. Pasquinucci, N. J. Cerf, M. Dušek, N. Lütkenhaus, and M. Peev, "The security of practical quantum key distribution," Rev. Mod. Phys. 81, 1301 (2009).

6. H.-K. Lo, M. Curty, and K. Tamaki, "Secure quantum key distribution," Nat. Photon. 8, 595 (2014).

7. C. E. Shannon, "Communication theory of secrecy systems," Bell Syst. Tech. J. 28, 656 (1949).

8. B. Schneier and P. Sutherland, Applied Cryptography: Protocols, Algorithms, and Source Code in C (Wiley, 1995).

9. P. W. Shor, "Polynomial-time algorithms for prime factorization and discrete logarithms on a quantum computer," SIAM J. Comput. 26, 1484 (1997).

10. R. Raussendorf and H. J. Briegel, “A one-way quantum computer,” Phys. Rev. Lett. 86, 5188 (2001).

11. E. Diamanti, H.-K. Lo, B. Qi, and Z.-L. Yuan, "Practical challenges in quantum key distribution,” NPJ Quantum Inf. 2, 16025 (2016).

12. H. J. Kimble, “The quantum internet,” Nature 453, 1023 (2008).

13. S. Wehner, D. Elkouss, and R. Hanson, "Quantum internet: a vision for the road ahead," Science 362, eaam9288 (2018). 
14. J. Qiu, "Quantum communications leap out of the lab," Nature 508, 441 (2014).

15. L. Greenemeier, "Election fix? Switzerland tests quantum cryptography," https:// www.scientificamerican.com/article/swiss-test-quantum-cryptography/ (2007).

16. F.-X. Xu, W. Chen, S. Wang, Z.-Q. Yin, Y. Zhang, and Y. Liu, "Field experiment on a robust hierarchical metropolitan quantum cryptography network," Chin. Sci. Bull. 54, 2991 (2009).

17. S. Wang, W. Chen, Z.-Q. Yin, Y. Zhang, T. Zhang, and H.-W. Li, "Field test of wavelength-saving quantum key distribution network," Opt. Lett. 35, 14 (2010).

18. S. Wang, W. Chen, Z.-Q. Yin, H.-W. Li, D.-Y. He, and Y.-H. Li, "Field and long-term demonstration of a wide area quantum key distribution network," Opt. Express 22, 21739 (2014).

19. W. Chen, Z.-F. Han, T. Zhang, W. Hao, Z.-Q. Yin, and F.-X. Xu, "Field experiment on a "star type" metropolitan quantum key distribution network,” IEEE Photon. Technol. Lett. 21, 575 (2009).

20. S.-K. Liao, W.-Q. Cai, J. Handsteiner, B. Liu, J. Yin, and L. Zhang, "Satelliterelayed intercontinental quantum network," Phys. Rev. Lett. 120, 030501 (2018).

21. S. Pirandola, U. L. Andersen, L. Banchi, M. Berta, D. Bunandar, and R. Colbeck, "Advances in quantum cryptography," Adv. Opt. Photon. 12, 1012 (2020).

22. S. Slussarenko and G. J. Pryde, "Photonic quantum information processing: a concise review," Appl. Phys. Rev. 6, 041303 (2019).

23. W.-Y. Hwang, "Quantum key distribution with high loss toward global secure communication," Phys. Rev. Lett. 91, 057901 (2003).

24. X.-B. Wang, "Beating the photon-number-splitting attack in practical quantum cryptography," Phys. Rev. Lett. 94, 230503 (2005).

25. H.-K. Lo, X.-F. Ma, and K. Chen, "Decoy state quantum key distribution," Phys. Rev. Lett. 94, 230504 (2005).

26. S. L. Braunstein and S. Pirandola, "Side-channel-free quantum key distribution," Phys. Rev. Lett. 108, 130502 (2012).

27. H.-K. Lo, M. Curty, and B. Qi, "Measurement-device-independent quantum key distribution," Phys. Rev. Lett. 108, 130503 (2012).

28. T. Sasaki, Y. Yamamoto, and M. Koashi, "Practical quantum key distribution protocol without monitoring signal disturbance," Nature 509, 475 (2014).

29. S. Pirandola, R. Laurenza, C. Ottaviani, and L. Banchi, "Fundamental limits of repeaterless quantum commuications," Nat. Commn. 8, 15043 (2017).

30. M. Lucamarini, Z.-L. Yuan, J. Dynes, and A. J. Shields, "Overcoming the ratedistance limit of quantum key distribution without quantum repeaters," Nature 557, 400 (2018).

31. A. Boaron, G. Boso, D. Rusca, C. Vulliez, C. Autebert, M. Caloz, M. Perrenoud, G. Gras, F. Bussières, M.-J. Li, D. Nolan, A. Martin, and H. Zbinden, "Secure quantum key distribution over $421 \mathrm{~km}$ of optical fiber," Phys. Rev. Lett. 121, 190502 (2018).

32. J.-P. Chen, C. Zhang, Y. Liu, C. Jiang, W.-J. Zhang, X.-L. Hu, J.-Y. Guan, Z.-W. Yu, H. Xu, J. Lin, M.-J. Li, H. Chen, H. Li, L. You, Z. Wang, X.-B. Wang, Q. Zhang, and J.-W. Pan, "Sending-or-not-sending with independent lasers: secure twin-field quantum key distribution over $509 \mathrm{~km}$," Phys. Rev. Lett. 124, 070501 (2020).

33. C. Simon, "Towards a global quantum network," Nat. Photon. 11, 678 (2017).

34. S. Pirandola and S. L. Braunstein, "Unite to build a quantum Internet," Nature 532, 169 (2016).

35. C. H. Bennett, F. Bessette, G. Brassard, L. Salvail, and J. Smolin, "Experimental quantum cryptography," J. Cryptol. 5, 3 (1992).

36. W. T. Buttler, R. J. Hughes, P. G. Kwiat, S. K. Lamoreaux, G. G. Luther, G. L. Morgan, J. E. Nordholt, C. G. Peterson, and C. M. Simmons, "Practical free-space quantum key distribution over $1 \mathrm{~km}$," Phys. Rev. Lett. 81, 3283 (1998).

37. R. J. Hughes, J. E. Nordholt, D. Derkacs, and C. G. Peterson, "Practical freespace quantum key distribution over $10 \mathrm{~km}$ in daylight and at night," New J. Phys. 4, 43 (2002).

38. C. Kurtsiefer, P. Zarda, M. Halder, H. Weinfurter, P. M. Gorman, P. R. Tapster, and J. G. Rarity, "Quantum cryptography: a step towards global key distribution," Nature 419, 450 (2002).

39. T. Schmitt-Manderbach, H. Weier, M. Fürst, R. Ursin, F. Tiefenbacher, T. Scheidl, J. Perdigues, Z. Sodnik, C. Kurtsiefer, J. G. Rarity, A. Zeilinger, and H. Weinfurter, "Experimental demonstration of free-space decoy-state quantum key distribution over 144 km," Phys. Rev. Lett. 98, 010504 (2007).
40. J. Yin, Y. Cao, Y.-H. Li, S.-K. Liao, L. Zhang, J.-G. Ren, W.-Q. Cai, W.-Y. Liu, B. Li, H. Dai, G.-B. Li, Q.-M. Lu, Y.-H. Gong, Y. Xu, S.-L. Li, F.-Z. Li, Y.-Y. Yin, Z.-Q. Jiang, M. Li, J.-J. Jia, G. Ren, D. He, Y.-L. Zhou, X.-X. Zhang, N. Wang, X. Chang, Z.-C. Zhu, N.-L. Liu, Y.-A. Chen, C.-Y. Lu, R. Shu, C.-Z. Peng, J.-Y. Wang, and J.-W. Pan, "Satellite-based entanglement distribution over 1200 kilometers," Science 356, 1140 (2017).

41. S.-K. Liao, W.-Q. Cai, W.-Y. Liu, L. Zhang, Y. Li, J.-G. Ren, J. Yin, Q. Shen, Y. Cao, Z.-P. Li, F.-Z. Li, X.-W. Chen, L.-H. Sun, J.-J. Jia, J.-C. Wu, X.-J. Jiang, J.-F. Wang, Y.-M. Huang, Q. Wang, Y.-L. Zhou, L. Deng, T. Xi, L. Ma, T. Hu, Q. Zhang, Y.-A. Chen, N.-L. Liu, X.-B. Wang, Z.-C. Zhu, C.-Y. Lu, R. Shu, C.-Z. Peng, J.-Y. Wang, and J.-W. Pan, "Satellite-to-ground quantum key distribution," Nature 549, 43 (2017).

42. Y.-A. Chen, Q. Zhang, T.-Y. Chen, W.-Q. Cai, S.-K. Liao, J. Zhang, K. Chen, J. Yin, J.-G. Ren, Z. Chen, S.-L. Han, Q. Yu, K. Liang, F. Zhou, X. Yuan, M.-S. Zhao, T.-Y. Wang, X. Jiang, L. Zhang, W.-Y. Liu, Y. Li, Q. Shen, Y. Cao, C.-Y. Lu, R. Shu, J.-Y. Wang, L. Li, N.-L. Liu, F. Xu, X.-B. Wang, C.-Z. Peng, and J.-W. Pan, "An integrated space-to-ground quantum communication network over 4600 kilometers," Nature 589, 214 (2021).

43. F. Fidler, M. Knapek, J. Horwath, and W. R. Leeb, "Optical communications for high-altitude platforms,” IEEE J. Sel. Top. Quantum Electron. 16, 1058 (2010).

44. S. Nauerth, F. Moll, M. Rau, C. Fuchs, J. Horwath, S. Frick, and H. Weinfurter, "Air-to-ground quantum communication," Nat. Photon. 7, 382 (2013).

45. C. J. Pugh, S. Kaiser, J. P. Bourgoin, J. Jin, N. Sultana, S. Agne, E. Anisimova, V. Makarov, E. Choi, and B. L. Higgins, "Airborne demonstration of a quantum key distribution receiver payload," Quantum Sci. Technol. 2, 024009 (2017).

46. J.-Y. Wang, B. Yang, S.-K. Liao, L. Zhang, Q. Shen, X.-F. Hu, J.-C. Wu, S.-J. Yang, H. Jiang, Y.-L. Tang, B. Zhong, H. Liang, W.-Y. Liu, Y.-H. Hu, Y.-M. Huang, B. Qi, J.-G. Ren, G.-S. Pan, J. Yin, J.-J. Jia, Y.-A. Chen, K. Chen, C.-Z. Peng, and J.-W. Pan, "Direct and full-scale experimental verifications towards ground-satellite quantum key distribution," Nat. Photon. 7, 387 (2013).

47. Y. Chu, R. Donaldson, R. Kumar, and D. Grace, "Feasibility of quantum key distribution from high altitude platforms," arXiv:2012.07479 (2020)

48. H.-Y. Liu, X.-H. Tian, C.-S. Gu, P.-F. Fan, X. Ni, R. Yang, J.-N. Zhang, M.-Z. Hu, J. Guo, X. Cao, X. Hu, G. Zhao, Y.-Q. Lu, Y.-X. Gong, Z. Xie, and S.-N. Zhu, "Drone based quantum entanglement distribution towards mobile quantum network," Natl. Sci. Rev. 7, 921 (2020).

49. H.-Y. Liu, X.-H. Tian, C.-S. Gu, P.-F. Fan, X. Ni, R. Yang, J.-N. Zhang, M.-Z. Hu, J. Guo, X. Cao, X. Hu, G. Zhao, Y.-Q. Lu, Y.-X. Gong, Z. Xie, and S.-N. Zhu, "Optical-relayed entanglement distribution using drones as mobile nodes," Phys. Rev. Lett. 126, 020503 (2021).

50. M. Zhang, L. Zhang, J.-C. Wu, S.-J. Yang, X. Wan, Z.-P. He, J.-J. Jia, D. S. Citrin, and J.-Y. Wang, "Detection and compensation of basis deviation in satellite-to-ground quantum communications," Opt. Express 22, 9871 (2014).

51. A. D. Hill, J. Chapman, K. Herndon, C. Chopp, D. J. Gauthier, and P. Kwiat, "Drone-based quantum key distribution," Urbana 51, 61801 (2017).

52. A. Conrad, S. Isaac, R. Cochran, D. Sanchez-Rosales, B. Wilens, A. Gutha, T. Rezaei, D. J. Gauthier, and P. Kwiat, "Drone-based quantum key distribution: QKD," Proc. SPIE 11678, 116780X (2021).

53. A. D. Hill, "Spatial mode control and advanced methods for multi-platform quantum communication," PhD. Thesis (University of Illinois at UrbanaChampaign, 2018).

54. J. P. Bourgoin, B. L. Higgins, N. Gigov, C. Holloway, C. J. Pugh, S. Kaiser, M. Cranmer, and T. Jennewein, "Free-space quantum key distribution to a moving receiver," Opt. Express 23, 33437 (2015).

55. H. Shakhatreh, A. H. Sawalmeh, A. Al-Fuqaha, Z.-C. Dou, E. Almaita, and I. Khalil, "Unmanned aerial vehicles (UAVs): a survey on civil applications and key research challenges," IEEE Access 7, 48572 (2019).

56. B. Li, Z.-S. Fei, Y. Zhang, and M. Guizani, "Secure UAV communication networks over 5G," IEEE Wireless Commun. 26, 114 (2019).

57. B. Qi, H.-K. Lo, C. C. W. Lim, G. Siopsis, E. A. Chitambar, R. Pooser, P. G. Evans, and W. Grics, "Free-space reconfigurable quantum key distribution network," in 2015 IEEE International Conference on Space Optical Systems and Applications (ICSOS) (IEEE, 2015), p. 1.

58. D. Vasylyev, A. A. Semenov, and W. Vogel, "Free-space quantum links under diverse weather conditions," Phys. Rev. A 96, 043856 (2017). 
59. C. Quintana, P. Sibson, G. Erry, Y. Thueux, E. Kingston, T. Ismail, and G. Faulkner, "Low size, weight and power quantum key distribution system for small form unmanned aerial vehicles," Proc. SPIE 10910, 1091014 (2019).

60. C. Agnesi, F. Vedovato, M. Schiavon, D. Dequal, L. Calderaro, M. Tomasin, D. G. Marangon, A. Stanco, V. Luceri, G. Bianco, G. Vallone, and P. Villoresi, "Exploring the boundaries of quantum mechanics: advances in satellite quantum communications," Trans. Royal Soc. A 376, 20170461 (2018).

61. D. Rideout, T. Jennewein, G. A. Camelia, T. F. Demarie, B. L. Higgins, and A. Kempf, "Fundamental quantum optics experiments conceivable with satellites-reaching relativistic distances and velocities, class," Quantum Grav. 29, 224011 (2012).

62. R. Ursin, T. Jennewein, J. Kofler, J. M. Perdigues, L. Cacciapuoti, C. J. de Matos, and M. Aspelmeyer, "Space-quest, experiments with quantum entanglement in space," Europhys. News 40, 26 (2009).

63. S. K. Joshi, J. Pienaar, T. C. Ralp, L. Cacciapuoti, W. McCutcheon, J. Rarity, D. Giggenbach, and J. G. Lim, "Space QUEST mission proposal: experimentally testing decoherence due to gravity," New J. Phys. 20, 063016 (2018).

64. R. Bedington, J. M. Arrazola, and A. Ling, "Progress in satellite quantum key distribution," NPJ Quantum Inf. 3, 30 (2017).

65. M. J. García-Martínez, N. Denisenko, D. Soto, D. Arroyo, A. B. Orue, and V. Fernandez, "High-speed free-space quantum key distribution system for urban daylight applications,” Appl. Opt. 52, 3311 (2013).

66. Y. Kaymak, R. Rojas-Cessa, J. Feng, N. Ansari, M.-C. Zhou, and T.-R. Zhang, "A survey on acquisition, tracking, and pointing mechanisms for mobile free-space optical communications," IEEE Commun. Surv. Tutor. 20, 1104 (2018).

67. Y. Saito, H. Takenaka, K. Shiratama, Y. Munemasa, A. Carrasco-Casado, P. V. Trinh, K. Suzuki, T. Fuse, Y. Takahashi, T. Kubo-oka, and M. Toyoshima, "Research and development of a transportable optical ground station in NICT: the results of the first performance test," Front Phys-Lausanne 9, 84 (2021).

68. F. Marsili, V. B. Verma, J. A. Stern, S. Harrington, A. E. Lita, T. Gerrits, I. Vayshenker, B. Baek, M. D. Shaw, R. P. Mirin, and S. W. Nam, "Detecting single infrared photons with 93\% system efficiency," Nat. Photon. 7, 210 (2013).

69. P. Hu, H. Li, L.-X. You, H.-Q. Wang, Y. Xiao, J. Huang, X.-Y. Yang, W.-J. Zhang, Z. Wang, and X.-M. Xie, "Detecting single infrared photons toward optimal system detection efficiency," Opt. Express 28, 36884 (2020).

70. H. Takenaka, A. Carrasco-Casado, M. Fujiwara, M. Kitamura, M. Sasaki, and M. Toyoshima, "Satellite-to-ground quantum-limited communication using a 50-kg-class microsatellite," Nature Photon. 11, 502 (2017).

71. D. Vasylyev and W. Vogel, "Satellite-mediated quantum atmosphere links," Phy. Rev. A 99, 053830 (2019).

72. J. P. Bourgoin, E. Meyer-Scott, B. L. Higgin, B. Helou, C. Erven, H. Hübel, B. Kumar, D. Hudson, I. D'Souza, R. Girard, R. Laflamme, and T. Jennewein, "A comprehensive design and performance analysis of low Earth orbit satellite quantum communication," New J. Phys. 15, 023006 (2013).

73. F. Moll, T. Botter, C. Marquardt, D. Pusey, A. Shrestha, A. Reeves, K. Jaksch, K. Gunthner, O. Bayraktar, and C. Mueller-Hirschkorn, "Stratospheric QKD: feasibility analysis and free-space optics system concept," Proc. SPIE 11167, 111670H (2019).

74. S. Bloom, E. Korevaar, J. Schuster, and H. Willebrand, "Understanding the performance of free-space optics," J. Opt. Netw. 2, 178 (2003).

75. M. Aspelmeyer, T. Jennewein, and A. Zeilinger, "Long-distance quantum communication with entangled photons using satellites," IEEE J. Sel. Top. Quantum Electron. 9, 1541 (2003).

76. M. Sasaki, "Quantum networks: where should we be heading," Quantum Sci. Technol. 2, 020501 (2017).

77. R. Ramanathan and J. Red, "A brief overview of ad hoc networks: challenges and directions," IEEE Commun Mag. 40, 20 (2002).

78. P. Senellart, G. Solomon, and A. White, "High-performance semiconductor quantum-dot single-photon sources," Nat. Nanotechnol. 12, 1026 (2017).

79. L. A. Ngah, O. Alibart, L. Labonté, V. D’Auria, and S. Tanzilli, "Ultra-fast heralded single photon source based on telecom technology," Laser Photon. Rev. 9, L1 (2015).

80. Y. Zhao, B. Qi, and H.-K. Lo, "Experimental quantum key distribution with active phase randomization," Appl. Phys. Lett. 90, 044106 (2007).
81. S.-H. Sun and L.-M. Liang, "Experimental demonstration of an active phase randomization and monitor module for quantum key distribution," Appl. Phys. Lett. 101, 071107 (2012).

82. X. Han, H.-L. Yong, P. Xu, K.-X. Yang, S.-L. Li, W.-Y. Wang, H.-J. Xue, F.-Z. Li, J.-G. Ren, C.-Z. Peng, and J.-W. Pan, "Polarization design for ground-to-satellite quantum entanglement distribution," Opt. Express 28, 369 (2020).

83. C. Z. Peng, J. Zhang, D. Yang, W.-B. Gao, H.-X. Ma, H. Yin, H.-P. Zeng, T. Yang, X.-B. Wang, and J.-W. Pan, "Experimental long-distance decoystate quantum key distribution based on polarization encoding," Phys. Rev. Lett. 98, 010505 (2007).

84. S. Nauerth, M. Furst, T. Schmitt-Manderbach, W. Henning, and W. Harald, "Information leakage via side channels in freespace BB84 quantum cryptography," New J. Phys. 11, 065001 (2009).

85. H. Ko, B.-S. Choi, J.-S. Choe, K.-J. Lim, J.-H. Kim, and C. J. Youn, "Highspeed and high-performance polarization-based quantum key distribution system without side channel effects caused by multiple lasers," Photon. Res. 6, 214 (2018).

86. M. Jofre, A. Gardelein, G. Anzolin, G. Molina-Terriza, J. P. Torres, M. W. Mitchell, and V. Pruneri, "100 MHz amplitude and polarization modulated optical source for free-space quantum key distribution at 850 nm," J. Lightwave Technol. 28, 2572 (2010).

87. Z. Yan, E. Meyer-Scott, J.-P. Bourgoin, B. L. Higgins, N. Gigov, A. MacDonald, H. Hübel, and T. Jennewein, "Novel high-speed polarization source for decoy-state BB84 quantum key distribution over free space and satellite links," J. Lightwave Technol. 31, 1399 (2013).

88. C. Bonato, M. Aspelmeyer, T. Jennewein, C. Pernechele, P. Villoresi, and A. Zeilinger, "Influence of satellite motion on polarization qubits in a Space-Earth quantum communication link," Opt. Express 14, 10050 (2006).

89. C. Bonato, C. Pernechele, and P. Villoresi, "Influence of all-reflective optical systems in the transmission of polarization-encoded qubits," J. Opt. A $\mathbf{9}$, 899 (2007).

90. L. Zhang, J.-J. Jia, S.-K. Liao, G.-H. Wen, R. Shu, and J.-W. Pan, "Establishment and in-orbit test of optical link in satellite-to-ground quantum communication," Sci. China: Inf. Science 48, 1183 (2018).

91. A. Laing, V. Scarani, J. G. Rarity, and J.-L. O'Brien, "Reference-frameindependent quantum key distribution," Phys. Rev. A 82, 012304 (2010).

92. Y. Xue, L. Shi, W. Chen, Z.-Q. Yin, G.-J. Fan, H.-Y. Fu, Q.-H. Lu, and J.-H. Wei, "Improving the performance of reference-frame-independent quantum key distribution through a turbulent atmosphere," Phys. Rev. A 102, 062602 (2020)

93. C. Agnesi, M. Avesani, L. Calderaro, A. Stanco, G. Foletto, M. Zahidy, A. Scriminich, F. Vedovato, G. Vallone, and P. Villoresi, "Simple quantum key distribution with qubit-based synchronization and a self-compensating polarization encoder," Optica 7, 284 (2020).

94. T. Ferreir, D. Vitoreti, G. B. Xavier, G. C. do Amaral, G. P. Temporão, and J. P. von der Weid, "Proof-of-principle demonstration of measurementdevice-independent quantum key distribution using polarization qubits," Phys. Rev. A 88, 052303 (2013).

95. J. C. Bienfang, A. J. Gross, A. Mink, B. J. Hershman, A. Nakassis, X. Tang, R. Lu, D. H. Su, C. W. Clark, and C. J. Williams, "Quantum key distribution with 1.25 Gbps clock synchronization," Opt. Express 12, 2011 (2004).

96. I. Marcikic, A. Lamas-Linares, and C. Kurtsiefer, "Free-space quantum key distribution with entangled photons," Appl. Phys. Lett. 89, 101122 (2006).

97. C.-Z. Peng, T. Yang, X.-H. Bao, J. Zhang, X.-M. Jin, F.-Y. Feng, B. Yang, J. Yang, J. Yin, Q. Zhang, N. Li, B.-L. Tian, and J.-W. Pan, "Experimental free-space distribution of entangled photon pairs over $13 \mathrm{~km}$ : towards satellite-based global quantum communication," Phys. Rev. Lett. 94, 150501 (2005).

98. R. Quan, Y.-W. Zhai, M.-M. Wang, F.-Y. Hou, S.-F. Wang, X. Xiang, T. Liu, S.-G. Zhang, and R.-F. Dong, "Demonstration of quantum synchronization based on second-order quantum coherence of entangled photons," Sci. Rep. 6, 30453 (2016)

99. A. Pljonkin, K. Rumyantsev, and P. K. Singh, "Synchronization in quantum key distribution," arXiv:1804.07003 (2018).

100. J.-G. Ren, J. Yin, B. Yang, F. Zhou, Z.-H. Yi, C.-Z. Peng, R. Shu, and J.-Y. Wang, "Time synchronization for quantum key distribution form ground to satellite," J. Infrared Millim. Terahertz Waves 30, 381 (2011).

101. N. M. P. Neumann, M. P. P. Heesch, and P. Graaf, "Quantum communication for military applications," arXiv:2011.04989 (2020) 
102. M. Krelina, "Quantum warfare: definitions, overview and challenges," arXiv:2103.12548 (2021).

103. Y. Zeng, R. Zhang, and T. J. Lim, "Wireless communications with unmanned aerial vehicles: opportunities and challenges," IEEE Commun. Mag. 54, 36 (2016).

104. S.-K. Liao, J. Lin, J.-G. Ren, W.-Y. Liu, J. Qiang, J. Yin, Y. Li, Q. Shen, L. Zhang, X.-F. Liang, H.-L. Yong, F.-Z. Li, Y.-Y. Yin, Y. Cao, W.-Q. Cai, W.-Z. Zhang, J.-J. Jia, J.-C. Wu, X.-W. Chen, S.-C. Zhang, X.-J. Jiang, J.-F. Wang, Y.-M. Huang, Q. Wang, L. Ma, L. Li, G.-S. Pan, Q. Zhang, Y.-A. Chen, C.-Y. Lu, N.-L. Liu, X. Ma, R. Shu, C.-Z. Peng, J.-Y. Wang, and J.-W. Pan, "Space-to-ground quantum key distribution using a smallsized payload on Tiangong-2 space lab," Chin. Phys. Lett. 34, 090302 (2017).

105. H. Chun, I. Choi, G. Faulkner, L. Clarke, B. Barber, G. George, C. Capon, A. Niskanen, J. Wabnig, D. O’Brien, and D. Bitauld, "Handheld free space quantum key distribution with dynamic motion compensation," Opt. Express 25, 6784 (2017).
106. F.-X. Wang, W. Wang, R. Niu, X.-Y. Wang, C.-L. Zou, C.-H. Dong, B. E. Little, S. T. Chu, H. Liu, P.-L. Hao, S.-F. Liu, and S. Wang, "Quantum key distribution with on-chip dissipative Kerr soliton," Laser Photon. Rev. 14, 1900190 (2020).

107. P. Sibson, C. Erven, M. Godfrey, S. Miki, T. Yamashita, M. Fujiwara, and M. Sasaki, “Chip-based quantum key distribution,” Nat. Commun. 8, 13984 (2017).

108. X. Zhang, B. A. Bell, A. Mahendra, C.-L. Xiong, P. H. W. Leong, and B. J. Eggleton, "Integrated silicon nitride time-bin entanglement circuits," Opt. Lett. 43, 3469 (2018).

109. Q. Gao, S.-H. Yi, Z.-F. Jiang, L. He, and X.-H. Wang, "Structure of the refractive index distribution of the supersonic turbulent boundary layer," Opt. Laser. Eng. 51, 1113 (2013).

110. J. P. Reid, A. K. Bertram, D. O. Topping, A. Laskin, S. T. Martin, M. D. Petters, F. D. Pope, and G. Rovelli, “The viscosity of atmospherically relevant organic particles," Nat. Commun. 9, 956 (2018). 